\title{
Unbestimmtheit im Vorfeld. Deutsche und norwegische Wikipedia-Artikel im Vergleich
}

\begin{abstract}
Der Beitrag befasst sich mit indefiniten Nominalphrasen im Vorfeld (d.h. in der Position vor dem finiten Verb in Aussagesätzen) in deutschen und norwegischen Wikipedia-Artikeln. Verglichen wird einerseits (quantitativ) die relative Häufigkeit indefiniter Nominalphrasen unterschiedlicher Struktur, andererseits (qualitativ) die (textbezogenen) informationsstrukturellen Bedingungen, unter denen solche Nominalphrasen typisch auftreten; dabei liegt das Hauptgewicht auf der qualitativen Untersuchung.
\end{abstract}

Abstract (norw.): Artikkelen behandler ubestemte nominalfraser i det såkalte forfeltet eller fundamentfeltet (dvs. plassen foran det finitte verbet i deklarativsetninger) i tyske og norske Wikipedia-artikler. Det dreier seg dels om en (kvantitativ) sammenlikning av hyppigheten forskjellige typer ubestemte nominalfraser, dels om en (kvalitativ) undersøkelse av de (diskursrelaterte) informasjonsstrukturelle betingelsene for forekomsten av slike nominalfraser. Hovedvekten ligger på det kvalitative aspektet.

\section{Einleitung}

Deutsch und Norwegisch sind beide sog. Verbzweitsprachen, d.h., das finite Verb steht im Aussagesatz(typ) an der zweiten Stelle, nach genau einem Satzglied, das seinerseits (fast) beliebiger Art sein kann; s. jeweils Zifonun/Hoffmann/Strecker (1997: 163) (stellvertretend für viele) und etwa Faarlund/Lie/Vannebo (1997). ${ }^{1}$ Die Position vor dem finiten Verb wird hier im Anschluss an die deutsche Grammatiktradition das Vorfeld genannt. Die Vorfeldbesetzung scheint in den beiden Sprachen auch grundsätzlich den gleichen, auf einem komplizierten Zusammenspiel zwischen Syntax, Semantik, Pragmatik und Informationsstruktur-Prosodie basierenden Prinzipien zu folgen (s. ProGr@mm kontrastiv ${ }^{2}$ ). Unterschiede bezüglich der Vorfeldbesetzung sind demzufolge eher im präferenziellen oder tendenziel-

1 Ich sehe hier von der Möglichkeit so genannter Mehrfachbesetzung ab; s. dazu Bassola/ Schwinn (in diesem Band) mit weiteren Hinweisen.

2 http://hypermedia.ids-mannheim.de/call/public/gruwi.ansicht?v_typ=0\&v_id=5885 (Stand: 9.2.2015). 
len Bereich zu verzeichnen bzw. zu erwarten. Beispielsweise scheint das Vorfeld im Norwegischen noch häufiger mit einem - eventuell expletiven - Subjekt und seltener mit einem adverbialen Satzglied besetzt zu werden, als es im Deutschen der Fall ist (Fabricius-Hansen/Solfjeld 1994; Haukås/Hoheisel 2013).

Im vorliegenden Aufsatz geht es um die Frage, unter welchen Bedingungen und in welchem Umfang indefinite Nominalphrasen im Vorfeld - oder genauer: im „linken Feld“ (Augustin, in diesem Band; s. Abschnitt 2) deutscher und norwegischer Wikipedia-Texte auftreten. Dabei beschränke ich mich auf indefinite Nominalphrasen im Singular (weiterhin: ISDPs), da diese in beiden Sprachen im Unterschied zu indefiniten Nominalphrasen im Plural mit einem expliziten unbestimmten Artikel - dt. ein(-), no. genuspezifisch en/ei/et - eingeleitet werden und folglich für die automatische Suche geeignet sind. Als Grundlage der Untersuchung dienen deutsche und norwegische Wikipedia-Artikel der aus WikipediaTexten von 2011 bestehenden EuroGr@mm-Korpora (Stand 2012). ${ }^{3}$ Für die einschlägigen Teilkorpora werden im Folgenden die Abkürzungen Wiki-DE und Wiki-NO verwendet.

Als Nullhypothese(n) bezüglich der Vorfeldbesetzung in Wiki-DE und WikiNO wäre anzunehmen, dass sie den allgemeinen Tendenzen in nicht-literarischen Prosatexten der jeweiligen Sprache folgt (HO) und dass der Anteil satzinitialer ${ }^{4}$ ISDPs an den nominal besetzten Vorfeldern dabei in etwa der gleiche ist (H1). Allerdings gibt es, wie ich an anderer Stelle (Fabricius-Hansen 2015) dargelegt habe, Gründe für die Annahme, dass Norwegisch stärker als Deutsch dazu neigt, nicht generisch interpretierte Indefinita (s. weiter unten, Abschnitt 4) in Subjektfunktion und dementsprechend auch als Vorfeldsubjekte $\mathrm{zu}$ vermeiden (vgl. Sveen 1996: 140ff.). In der Praxis würde das heißen, dass im No. öfter als im Dt. unter sonst gleichen kontextuellen Bedingungen eine Ausdrucksalternative ohne ISDP-Subjekt gewählt wird:

i. Diatheseveränderung, d.h. beispielsweise ein Passivsatz mit indefiniter Agensphrase statt eines transitiven Aktivsatzes mit indefinitem Subjekt (Pitz 2006);

ii. eine sog. Präsentierungskonstruktion, in der det 'es' als expletives strukturelles Subjekt eines intransitiven Verbs erscheint, während das 'eigentliche'

3 Die damalige Version des norwegischen Wikipedia-Korpus enthielt allerdings auch Diskussionsbeiträge, die inzwischen (2014) aussortiert worden sind. Für genauere Informationen sei auf www.ids-mannheim.de/cosmas2/ verwiesen.

4 Die Ausdrücke satzinitial und im Vorfeld (stehend) werden hier und im Folgenden als Synonyme verwendet. 
(indefinite) Subjekt in der strukturellen Objektposition erscheint (Askedal 1986; Faarlund/Lie/Vannebo 1997: 827ff.; Sveen 1996); ${ }^{5}$

III. ein Spaltsatz, in dem die indefinite Nominalphrase als prädikativische „CleftKonstituente“ dient, als Alternative zu einem Satz mit eng fokussiertem indefinitem Subjekt (vgl. (Engebretsen 2012; Faarlund/Lie/Vannebo 1997: 1088ff.).

Man vergleiche dazu jeweils die Satzpaare (1)/(2), (3)/(4) und (5)/(6), die dem Oslo Multilingual Corpus $\left(\mathrm{OMC}^{6}\right)$ entnommen sind. (Die einschlägigen ISDPs sind unterstrichen.)

(1) Um mich herum ist nur Finsternis, ich kann die Hand nicht vor Augen sehen. Auf nackten Zehen schleiche ich mich zur Tür, öffne sie und blicke hinaus. Ein blauweißes Licht zerreißt die schwarze afrikanische Sommernacht. (TB1TD)

(2) [...] Den afrikanske svarte sommernatta flerres av et blåhvitt lys et stykke borte. (TB1)

Lit.: Die afrikanische schwarze Sommernacht wird-zerrissen von einem blauweißen Licht ein Stück entfernt.

(3) Eines Tages aber geschah etwas, das eine Bedrohung für ihr Glück war. Ein Fremder kam in die Stadt, der sich in der freien, der südlichen Ecke des Marktplatzes niederließ. (JW1TD)

(4) Men en dag skjedde det noe som truet deres lykke. Det kom en fremmed til byen. Han slo seg ned i markedsplassens ledige hjørne, det mot syd. (JW1) Lit.: [...] Es kam ein Fremder in die Stadt. Er ließ sich nieder in des Marktplatzes freier Ecke, der gegen Süden.

(5) Ich erzählte ihm die Legende, die ich selbst wenige Monate zuvor im Gasthaus gehört hatte. Ein Kaufmann, der aus der Stadt jenseits der Berge kam, erzählte sie mir. (JW1TD)

5 Nach Sveen (1996: 140ff.) dient die Präsentierungskonstruktion dazu, eine mit der Subjektposition verbundene generische oder spezifische Lesart der indefiniten Nominalphrase auszuschalten. Allerdings scheint er den - notorisch unklaren - Terminus „spezifisch“ in einem etwas engeren Sinne zu verstehen, als hier vorgeschlagen wurde.

6 Siehe www.hf.uio.no/ilos/english/services/omc/ (Stand: 9.2.2015); die jeweilige Übersetzung ist als TN bzw. TD markiert. 
(6) Jeg fortalte ham den legenden jeg selv hørte i vertshuset bare for noen måneder siden. Det var en handelsmann som fortalte den. Han kom fra byen på den andre siden av fjellene. (JW1)

Lit.: [...] Es war ein Kaufmann, der sie erzählte. Er kam aus der Stadt auf der anderen Seite der Berge.

Aufschlussreich ist in dieser Hinsicht auch das Paar (7)/(8), wo der einschlägige Originalsatz in (7) ein transitives Verb mit spezifisch referierendem ISDP-Subjekt und ISDP-Objekt aufweist und Passivierung in der Übersetzung deshalb nichts bringen würde. Die Präsentierungsalternative wird andererseits durch die transitive Konstruktion blockiert, und ein Spaltsatz ist aus informationsstrukturellen Gründen nicht aktuell. In der Übersetzung (8) ist nun ein intransitives (sog. 'ergatives') Pendant zu etwas bringen, nämlich komme med noe 'mit etwas kommen', gewählt worden, das es ermöglicht, die indefinite Subjektübersetzung en lastebil ,ein Lastwagen' via Präsentierung in der Objektposition, vor der präpositional angeschlossenen indefiniten Objektübersetzung et lass klær 'eine Ladung Kleider', unterzubringen.

(7) Ein Lastwagen hatte aus Linz eine Ladung Kleider gebracht. Sie waren in einer Baracke abgeladen worden. (SW1)

(8) Det var kommet en lastebil fra Linz med et lass klær. De var blitt lagt i en brakke. (SW1TN)

Lit.: Es war gekommen ein Lastwagen aus Linz mit einer Ladung Kleider. Sie waren worden (ab)gelegt in einer Baracke.

Vor diesem Hintergrund könnte die folgende Hypothese, alles Andere gleich, plausibler erscheinen als die oben angedeutete kontrastive Nullhypothese H1.

H1' ISDPs haben in Wiki-DE einen größeren Anteil an den nominal besetzten Vorfeldern als in Wiki-NO.

Allerdings können wir eben nicht davon ausgehen, dass alles Andere gleich ist oder dass HO zutrifft. Zum einen scheinen Wikipedia-Texte eine eigene Textsorte zu bilden, die u.A. im Hinblick auf die Vorfeldbesetzung nicht unbedingt mit sonst vergleichbaren Textsorten übereinstimmt (s. Augustin in diesem Band); vor allem ist mehr oder weniger massive (inhaltliche und) sprachliche Beeinflussung durch englischsprachige Wikipedia-Texte nicht auszuschließen. Um das zu überprüfen, müssten Wiki-DE und Wiki-NO einerseits mit anderen dt. bzw. no. Texten ähnlicher Gattung und andererseits mit englischen Wikipedia-Texten verglichen werden. Zum anderen könnten Artikeltypen, die das Referieren auf 'partikuläre' 
Ereignisse/Sachverhalte und Einzelindividuen/-objekte favorisieren, und Artikeltypen, die eher auf Verallgemeinerungen abzielen, in Wiki-DE und Wiki-NO ungleich vertreten sein. Eine Verifizierung oder Falsifizierung von H1 würde mithin keine Schlüsse erlauben bezüglich unterschiedlicher sprachlicher Präferenzen. Die Stärke der mutmaßlichen 'Abneigung' gegenüber satzinitialer Indefinitheit müsste schließlich für beide Vergleichssprachen unter Heranziehung der Alternativen ermittelt werden - und für eine quantitative Untersuchung dieser Art, bei der es nicht nur um das linke Feld geht, ist das Wikipedia-Korpus des IDS in seiner jetzigen Form nicht ganz geeignet, da die Annotierung über die Ebene der Wortarten (PoS) nicht hinausgeht. ${ }^{7}$

Aus diesen und verwandten Gründen wird hier auf tief gehende quantitative Analysen verzichtet. Nach einer kurzen, weitgehend auf Augustin (in diesem Band) basierenden Präsentation einiger quantitativer Eckdaten zum (ISDP-Vorkommen im) linken Feld in den deutschen und norwegischen Wikipedia-Korpora des IDS (Abschnitt 2) soll vielmehr der Versuch unternommen werden, anhand von jeweils zwei Stichproben von jeweils etwa 1.000 Belegen aus Wiki-DE und Wiki-NO typische (syntaktische, semantisch-pragmatische, informationsstrukturelle) Vorkommensbedingungen satzinitialer ISDPs zu identifizieren und miteinander zu vergleichen (Abschnitt 3 bis 5). Um maximale Vergleichbarkeit $\mathrm{zu}$ sichern, beschränke ich mich dabei auf ISDPs, die aus zwei bzw. drei Wörtern bestehen; die Einbeziehung umfangreicherer Vorfeld-ISDPs wäre natürlich wünschenswert, konnte aber im Rahmen dieser Arbeit nicht geleistet werden. Im abschließenden Abschnitt 6 werden die Ergebnisse zusammengefasst.

\section{Ein-, zwei- und dreiwortige „linke Felder“ in Wiki-DE und Wiki-NO}

Zunächst ein paar Worte über die beiden Wiki-Korpora: Gemessen an der Wortzahl ist Wiki-DE fast achtmal größer als Wiki-NO (551,09 gegenüber 70,38 Millionen Wörter). Was die Gesamtzahl der durch die Suchanfragen (s. Augustin in diesem Band) erfassten „linken Felder“ betrifft, ist der Unterschied noch größer: 19.445.906 (Wiki-DE) gegenüber 1.743.976 (Wiki-NO), d.h., in Wiki-DE wurden gut zehnmal so viele linke Felder registriert wie in Wiki-NO. Unter dem linken Feld

7 Dass det-Konstruktionen der einen oder der anderen Art auch in Wiki-NO eine wichtige Rolle spielen, kann allerdings nicht bezweifelt werden: Nach Augustin (in diesem Band) ist det 'es' das zweithäufigste Wort in Ein-Wort-linken-Feldern in Wiki-NO. 
einer zwischen zwei Satzgrenzen stehenden (Satz-)Einheit ist nach Augustin der Textbereich zwischen der linken Satzgrenze und der ersten nachfolgenden finiten Verbform zu verstehen. ${ }^{8}$ Die entsprechenden Sätze/Satzeinheiten haben mithin eine durchschnittliche Länge von jeweils knapp 30 Wörtern in Wiki-DE und gut 40 Wörtern in Wiki-NO. Obwohl der Durchschnittswert für Wiki-DE über dem errechneten Durchschnittswert für 'lange' Sätze des Gegenwartsdeutschen (s. etwa Braun 1998; Glück/Sauer 1997) liegt, scheint das Ergebnis jedoch grundsätzlich mit bisherigen Untersuchungen zur Satzlänge im Deutschen verträglich. Es reimt sich aber schlecht mit vergleichenden Korpusanalysen, denen zufolge Sätze im Norwegischen tendenziell kürzer - oder auf jeden Fall nicht länger - sind als im Deutschen (Fabricius-Hansen/Solfjeld 1994; Ramm 2011). Die Diskrepanz könnte darauf hindeuten, dass die Suchanfrage in Wiki-DE häufiger als in WikiNO einfache V2-Sätze und nicht etwa Satzreihen erfasst - oder dass die POSAnnotation in Wiki-NO nicht ganz angemessen ist. Bei linken Feldern, die aus einem, zwei oder drei Wörtern bestehen $\left(\mathrm{LF}_{1}, \mathrm{LF}_{2}\right.$ und $\left.\mathrm{LF}_{3}\right)$, dürfen wir jedoch davon ausgehen, dass sie im Wesentlichen traditionellen Vorfeldern entsprechen.

Tabelle 1 zeigt die relative Häufigkeit von linken Feldern mit bis zu fünf Wörtern; allgemein gilt, dass die Häufigkeit eines LF-Typs in beiden Korpora mit dessen Umfang abnimmt (Augustin in diesem Band). Ein-, zwei- und dreiwortige linke Felder sind mithin in beiden Korpora die ranghäufigsten LF-Typen. Dass LF die Hälfte aller LF-Vorkommen in Wiki-NO ausmachen gegenüber knapp einem Drittel (30,8\%) in Wiki-DE, hängt zweifellos zum Teil damit zusammen, dass unattribuierte definite Nominalphrasen im Norwegischen aus einem Wort (Substantiv mit Artikelsuffix), im Deutschen hingegen aus zwei Wörtern bestehen; vgl. Augustin (in diesem Band). Wie aus der Tabelle hervorgeht, decken $\mathrm{LF}_{2}$ und $\mathrm{LF}_{3}$, auf die wir uns im nächsten Abschnitt konzentrieren, insgesamt jeweils 36,4\% (Wiki-DE) und 27,6\% (Wiki-NO) der LF-Vorkommen ab.

Tab. 1: Relative Häufigkeit von LF-Größen in Wiki-DE und Wiki-NO (nach Augustin in diesem Band, Tab. 5)

\begin{tabular}{llr}
\hline & Wiki-DE & Wiki-NO \\
\hline $\mathbf{L F}_{1}$ (Ein-Wort-Felder) & $30,8 \%$ & $50,1 \%$ \\
$\mathbf{L F}_{2}$ (Zwei-Wort-Felder) & $21,3 \%$ & $17,0 \%$ \\
$\mathbf{L F}_{3}$ (Drei-Wort-Felder) & $15,1 \%$ & $10,6 \%$ \\
\hline
\end{tabular}

8 Das heißt, das „linke Feld“ umfasst nicht nur das Vorfeld, sondern auch das sog. linke Außenfeld (oder Vor-Vorfeld), das jedoch in unserem Zusammenhang, wo es um „linke Felder“ mit zwei oder drei Wörtern geht, kaum Relevanz hat. 


\begin{tabular}{llr}
\hline & Wiki-DE & Wiki-NO \\
\hline $\mathbf{L F}_{4}$ (Vier-Wort-Felder) & $10,7 \%$ & $6,4 \%$ \\
$\mathbf{L F}_{5}$ (Fünf-Wort-Felder) & $4,3 \%$ & $2,8 \%$ \\
Insgesamt & $82,2 \%$ & $86,9 \%$ \\
\hline
\end{tabular}

Tabelle 2A zeigt die Häufigkeit satzinitialer unattribuierter ISDPs - d.h. die Häufigkeit von Vorfeldern, die aus ein(-) bzw. en/ei/et + Nomen bestehen - relativ zu Zwei-Wort-Feldern sowie Ein- und Zwei-Wort-Feldern insgesamt; LF ${ }_{1}$ wurde mit einbezogen, um der Tatsache Rechnung zu tragen, dass zweiwortigen definiten Nominalphrasen im Deutschen Ein-Wort-Phrasen im Norwegischen entsprechen (s.o.). In Tabelle 2B wird die absolute und relative Häufigkeit von satzinitialen Drei-Wort-ISDPs angegeben, die ein Adjektiv (i.w.S.) zwischen dem indefiniten Artikel und dem Kopfnomen enthalten. ${ }^{9}$

Tab. 2: Häufigkeit von Indefinitartikel-N bzw. Indefinitartikel-Adjektiv-N im $\left(\mathrm{LF}_{1}+\right) \mathrm{LF}_{2}$ bzw. $\mathrm{LF}_{3}$

\begin{tabular}{|c|c|c|c|c|c|c|c|}
\hline & & Wiki-DE & & & Wiki-NO & & \\
\hline \multirow{5}{*}{ A } & LF-Typ & \multicolumn{3}{|c|}{$\operatorname{ein}(-)+\mathrm{N}$} & \multirow[b]{2}{*}{ Anzahl } & \multicolumn{2}{|c|}{ en/ei/et $+\mathrm{N}$} \\
\hline & & Anzahl & Anzahl & $\%$ & & Anzahl & $\%$ \\
\hline & $\mathrm{LF}_{1}$ & 6.18641 & - & & 1.060 .448 & - & \\
\hline & $\mathrm{LF}_{2}$ & 4.458546 & 93.477 & $2,1 \%$ & 459.887 & 10.808 & $2,4 \%$ \\
\hline & $\mathrm{LF}_{1}$ und $\mathrm{LF}_{2}$ & 10.477 .187 & 93.477 & $0,9 \%$ & 1.520 .335 & 10.808 & $0,7 \%$ \\
\hline \multirow{3}{*}{ B } & & & \multicolumn{2}{|c|}{$\operatorname{ein}(-)+\operatorname{Adj}+\mathrm{N}$} & & \multicolumn{2}{|c|}{ en/ei/et + Adj +N } \\
\hline & & & Anzahl & $\%$ & & Anzahl & $\%$ \\
\hline & $\mathrm{LF}_{3}$ & 3.294 .346 & 77.763 & $2,4 \%$ & 276.996 & 5.114 & $1,8 \%$ \\
\hline
\end{tabular}

Aus der Tabelle geht hervor, dass Zwei-Wort-Vorfelder äußerst selten mit einer ISDP besetzt sind, und zwar etwas seltener in Wiki-DE als in Wiki-NO (2,1\% gegenüber 2,4\%); bezieht man aus den oben genannten Gründen die Ein-WortVorfelder mit ein, so reduziert sich der ISDP-Anteil auf weniger als ein Prozent und das Verhältnis zwischen Wiki-DE und Wiki-NO kehrt sich um $(0,9 \%$ gegenüber 0,7\%). Für die Häufigkeit pränominal attribuierter ISDPs in Drei-Wort-Vorfeldern ist der Unterschied zwischen den beiden Korpora etwas größer (2,4\% gegenüber 1,8\%). Nicht attribuierte ISDPs und nicht attribuierte definite Nominal-

9 Die einschlägigen Recherchen sowie die im Abschnitt 3 besprochene Stichprobenauswahl wurden von Hagen Augustin durchgeführt, dem ich hiermit herzlich danke. 
phrasen (im Singular oder Plural) kommen in Wiki-DE im Verhältnis 1:21, in WikiNO im Verhältnis 1:25 im Vorfeld vor (in absoluten Zahlen jeweils 93.477/1.969.139 und 10.808/264.827), d.h. etwas häufiger in Wiki-DE. Alles in allem scheinen die Ergebnisse, deren statistische Signifikanz allerdings nicht überprüft worden ist, mithin die kontrastive Hypothese H1' schwach zu unterstützen, ohne jedoch die alternative Nullhypothese $\mathrm{H} 1 \mathrm{zu}$ widerlegen; d.h., sie sind soweit mit beiden Hypothesen verträglich.

In den folgenden Abschnitten sollen die kontextuellen - syntaktischen, semantisch-pragmatischen, informationsstrukturellen - Bedingungen, unter denen zwei- und dreiwortige ISDPs in Wiki-DE und Wiki-NO satzinitial in nicht adverbialer Funktion anzutreffen sind, anhand von vergleichbaren, im Abschnitt 3 präsentierten Stichproben näher untersucht werden.

\section{Zweimal zwei Stichproben. Syntaktische Funktionen der Vorfeld-ISDPs}

Die den folgenden Ausführungen zugrunde liegenden Stichproben bestehen aus jeweils gut 1.000 Zufallstreffern für folgende Strukturen im linken Feld:

- Indefinitartikel + Substantiv (gekürzt weiterhin IndefN): eine Studie, einen Welterfolg; en studie, et solur 'Sonnenuhr'.

- Indefinitartikel + beliebiges Wort + Substantiv (IndefXN): ein vergleichbares Abkommen, einen neuen Aufschwung; en ny 'neu' leder 'Leiter', et annet 'ander-' bibelsted 'Bibelstelle'.

Unter den Treffern befanden sich auch einige nicht einschlägige Strukturen wie adverbial verwendete ISDPs und partitive/quantifizierende Konstruktionen; vgl. jeweils (9)-(10) und (11)-(12). Diese wurden manuell aussortiert.

(9) Eines Tages wird George mit mehr als $330 \mathrm{~kg}$ Marihuana erwischt und zu einer Haftstrafe verurteilt. (WPD11: Blow (Film))

(10) Et annet sted står det at [...].

Lit: Eine andere Stelle steht es dass [...].

'An einer anderen Stelle steht, dass [...].'

(11) Einer dieser Generatoren hatte sich noch während der Startphase des Value-Jets unbemerkt aktiviert. (WPD11: Douglas DC-9)

(12) En rekke småelefanter har blitt fanget og vist frem i dyrehager i New York og Antwerpen [...]. (WPN11: Pygméelefant) 
'Eine Reihe Kleinelefanten ist gefangen und in Tiergärten in New York und Antwerpen gezeigt worden.'

Erwünscht waren bei den Drei-Wort-Stichproben primär indefinite Nominalphrasen mit einem vorangestellten Adjektivattribut (vgl. Abschnitt 2), erfasst und beibehalten wurden dabei auch - vor allem in den NO-Stichproben ${ }^{10}$ - vereinzelte Strukturen anderer Art:

- Indefinitartikel + Substantiv im Genitiv + Substantiv: en gladiators liv 'eines Gladiators Leben',

- Indefinitartikel + Substantiv + nachgestellte nominale Apposition: en Abbacover „Fernando“.

Belege wie (13), wo die ambige Verbform ledet 'leitete(-)/geleitet' offensichtlich als finite Präteritumform statt als Partizip Perfekt registriert worden ist, wurden hingegen ausgeklammert, da das Vorfeld in solchen Fällen mehr als die gesuchte Anzahl Wörter enthält.

(13) Et utvalg ledet av Nordland fylkeskommune leverte sin rapport 25. januar 2007. (WPN11: Hangglider)

'Eine Kommission geleitet von Nordland Großgemeinde lieferte seinen Bericht 25. Januar 2007.'

Übrig blieben nach der Sichtung vier Stichproben von 800 bis knapp 1.000 Belegen: IndefN-DE, IndefXN-DE, IndefN-NO, IndefXN-NO.

Die Vorfeld-ISDPs kommen in den Stichproben als Subjekte, als Objekte und als Prädikative vor; vgl. (14)-(19). Die Frequenz der Funktionstypen wird in den Tabellen 3 und 4 gezeigt.

ISDP-Subjekt:

(14) Ein Sieg blieb dem Team, das zwei zweite Plätze als beste Resultate vorweisen konnte, verwehrt. (WPD11: Alain Prost)

(15) En prototype ble bygget i 1942, men [...]. (WPN11: Antonov An 40) 'Ein Prototyp wurde 1942 gebaut, aber [...]'

10 Der benutzte norwegische POS-Tagger hat sich leider als recht unzuverlässig erwiesen. 
ISDP-Objekt:

(16) 1966 politierte [sic!] er einen Formel-2-Brabham beim Großen Preis von Deutschland am Nürburgring. Einen „echten“ Formel-1-Wagen fuhr er 1967 in Silverstone. Mit dem Werks-Cooper T81 wurde er mit vier Runden Rückstand Neunter. WPD11/A42.82324: Alan Rees

(17) I 1955 ble hun tildelt en Bodil for sin innsats som moren i Altid Ballade. Et annet høydepunkt oppnådde hun i samspillet med Jørgen Ryg i TV-stykket Syg og munter. (WPN11/S04.86053: Sigrid Horne-Rasmussen)

'Im Jahr 1955 erhielt sie den Bodil-Preis für ihren Einsatz als die Mutter in Immer Krach. Einen anderen Höhepunkt erreichte sie im Zusammenspiel mit Jörgen Ryg im TV-Stück Krank und munter.'

ISDP-Prädikativ:

(18) Ein anderes Thema ist die Entstehung des Weinens und der Flecken im Mond sowie die Entstehung der Völker. (WPD11/A17.28103: Afrikanische Religionen)

(19) Et annet problem er paradoksalt nok faren for uttørking [...]. (WPN11: Sump)

'Ein anderes Problem ist paradoxerweise die Gefahr der Austrocknung $[\ldots]$

Tab. 3: Syntaktische Funktionen der Vorfeld-ISDPs - Wiki-DE-Stichproben

\begin{tabular}{lrrrrrr}
\hline Strukturtyp & IndefN & \multicolumn{3}{c}{ IndefXN } & \multicolumn{3}{c}{ Insg. Indef(X)N } \\
Funktion & Anzahl & $\%$ & Anzahl & $\%$ & Anzahl & $\%$ \\
\hline Subjekt & 791 & 81,9 & 585 & 61,4 & 1.376 & 71,7 \\
Objekt & 90 & 9,3 & 165 & 17,3 & 255 & 13,3 \\
Prädikativ & 85 & 8,8 & 203 & 21,3 & 288 & 15,0 \\
Insgesamt & 966 & 100 & 953 & 100 & 1.919 & 100 \\
\hline
\end{tabular}

Tab. 4: Syntaktische Funktionen der Vorfeld-ISDPs - Wiki-NO-Stichproben

\begin{tabular}{lrrcrrr}
\hline Strukturtyp & IndefN & \multicolumn{3}{c}{ IndefXN } & \multicolumn{3}{c}{ Insg. Indef(X)N } \\
Funktion & Anzahl & $\%$ & Anzahl & $\%$ & Anzahl & $\%$ \\
\hline Subjekt & 888 & 92,6 & 667 & 76,1 & 1.555 & 84,7 \\
Objekt & 2 & 0,2 & 8 & 0,9 & 10 & 0,5 \\
Prädikativ & 69 & 7,2 & 202 & 23,0 & 271 & 14,8 \\
Insgesamt & 959 & 100 & 877 & 100 & 1.625 & 100 \\
\hline
\end{tabular}


Aus den Tabellen geht Folgendes hervor:

(i) Die Subjektfunktion ist in beiden Stichprobenkorpora weitaus am stärksten vertreten, und zwar etwas stärker im norwegischen Korpus als im deutschen (93\% gegenüber 82\% im Zwei-Wort- und 85\% gegenüber 72\% im DreiWort-Fall).

(ii) Während ISDP-Prädikative und ISDP-Objekte in der Wiki-DE-Auswahl fast gleich häufig vorkommen, nehmen Prädikative in der Wiki-NO-Auswahl einen klaren zweiten Platz ein; die Objekte machen hier weniger als 1\% aus.

(iii) Subjekte kommen unter dreiwortigen (IndefXN) ISDPs etwas seltener - und Prädikative bzw. Prädikative und Objekte entsprechend häufiger - vor als unter den zweiwortigen (IndefN) (85\% gegenüber 93\% für Wiki-NO, 72\% gegenüber $82 \%$ für Wiki-DE).

$\mathrm{Zu}$ (i): Dass es sich bei den Vorfeld-ISDPs in Wiki-NO noch häufiger um Subjekte handelt als in Wiki-DE, entspricht unseren Erwartungen (s. Abschnitt 1). Zu (ii): Das Ergebnis steht im Einklang mit der Annahme (Fabricius-Hansen 2015), dass Nominalphrasen im Norwegischen aus morphosyntaktischen Gründen seltener als Vorfeldobjekte auftreten, als es im Deutschen der Fall ist. Zu (iii): Das unterschiedliche Verhältnis von Subjekt- und Prädikativ/Objekt-ISDPs in den IndefNund den IndefXN-Stichproben scheint informationsstrukturelle Gründe zu haben, auf die ich unten (Abschnitt 5) zu sprechen komme. Vorerst sollen die Stichproben jedoch im Hinblick auf die Verteilung verschiedener Interpretations- oder Lesartkategorien der ISDPs verglichen werden.

\section{Lesarten von Vorfeld-ISDPs in Subjekt- und Objektfunktion}

Die Interpretation indefiniter Nominalphrasen variiert bekanntlich erheblich nach dem Kontext (s. etwa Büring 2001; Endriss/Hinterwimmer 2009; Farkas 2002; Heim 2011; von Heusinger 2011). Das trifft leider auch auf die einschlägige linguistische Terminologie oder Begrifflichkeit zu. Sehen wir von der prädikativischen Verwendung ab, so genügt für unsere Zwecke die - zugegeben vereinfachende - Unterscheidung zwischen einer spezifisch referierenden oder existenziellen, einer (nicht-generalisierend) unspezifischen und einer generischen oder generalisierenden Verwendung (Lesart, Interpretation) der ISDPs (vgl. FabriciusHansen 2015).

Spezifisch referierende ISDPs (im Folgenden Spez-ISDP abgekürzt) liegen in Fällen wie (20)-(21) vor, wo die Nominalphrase einen neuen Referenten in den 
Diskurs einführt; repräsentiert man indefinite Nominalphrasen formal-semantisch mit Hilfe des Existenzoperators, so hat dieser in solchen Fällen den weitesten Skopus (Endriss/Hinterwimmer 2009; Heim 2011).

(20) Ein Magier betritt eine Bühne und verneigt sich. Anschließend lässt er seine Handschuhe, seinen Hut und seinen Mantel verschwinden. (WPD11: The Magician)

(21) BMW etablerte seg som flymotorprodusent under første verdenskrig. [...] En stor fabrikk ble bygd utenfor Münchens flyplass Oberwiesenfeld. [...] BMWs fabrikk i München ble bombet til ruiner i 1944-45 (WPN11: BMW) 'BMW etablierte sich im Ersten Weltkrieg als Flugmotorproduzent. [...] Eine große Fabrik wurde außerhalb von Münchens Flughafen Oberwiesenfeld gebaut. [...] BMWs Fabrik in München wurde 1944-45 zerbombt.'

Die Kategorie 'generisch oder generalisierend' (Gen-ISDP) umfasst teils „kind“bezogene „definitional generics“ (Krifka 2012), wie sie in (22)-(23) veranschaulicht werden, teils ISDPs die semantisch im Skopus eines impliziten generischen Operators oder eines expliziten generalisierenden Quantors wie immer, nie, oft, manchmal stehen („descriptive generalizations“ nach Krifka 2012), wie jeweils in (24) und (25)-(26).

(22) Ein Ernährungsteam ist ein Fachgremium für medizinische Ernährungsfragen in einem Krankenhaus. (WPD11: Ernährungsteam)

(23) En apoplektiker er en person som lider av apopleksi [...]. (WPN11: Apoplektiker)

'Ein Apoplektiker ist eine Person, die an Apoplexie leidet.'

(24) Die Brutdauer eines kleineren Singvogels wie der Kohlmeise beträgt zehn bis vierzehn Tage. Ein junger Schwan benötigt ungefähr 35 Tage bis zum Schlüpfen. (WPD11: Brutdauer)

(25) Geisha i dag er utelukkende kvinner. [...] En jente bruker ofte seks år på å bli en geisha, [...]. (WPN11/G03.34707: Geisha)

'Geisha sind heute ausschließlich Frauen. ... Ein Mädchen braucht oft sechs Jahre um eine Geisha zu werden.'

(26) En Horowitz-konsert var alltid in begivenhet. (WPN11/V00.10345: Vladimir Horowitz)

'Ein Horowitz-Konzert war immer ein Ereignis.' 
Als unspezifisch (Unspez-ISDP) bezeichne ich nicht-generalisierende Verwendungen, bei denen die ISDP bzw. der Existenzoperator sich semantisch im Skopus einer Negation oder Modaloperators oder in einem anderswie opaken Kontext befindet und folglich keinen Referenten im Diskursuniversum etabliert; vgl. (27)-(29).

(27) Grab 1 gehört zum Typ der Großdolmen. Ein Grabhügel lässt sich nicht ausmachen, eine Grabeinfassung ist heute nicht mehr vorhanden. Die Grabkammer ist nordwest-südöstlich orientiert. (WPD11: Großsteingräber bei Schadewohl)

(28) Boka “Løping. En verdenshistorie” er foreløpig oversatt til svensk, engelsk, russisk, nederlandsk, italiensk, amharisk, tysk, japansk, arabisk, portugisisk og koreansk. Den er under oversettelse til finsk, tyrkisk, tsjekkisk og slovakisk. En kinesisk utgave er utsatt som følge av Nobelpristildelingen 2010. (WPN11: Thor Gotaas)

'Das Buch „Laufen. Eine Weltgeschichte“ ist vorläufig ins Schwedische, [...] und Koreanische übersetzt worden. Es wird zur Zeit ins Finnische, [...] und Slowakische übersetzt. Eine chinesische Ausgabe ist als Folge der Nobelpreisverleihung 2010 hinausgeschoben worden.'

(29) Den 18. februar 1516 [...] fødte hun en datter som ble døpt Maria (Mary), den senere Maria I av England [...]. [...] En mannlig arving var særdeles viktig for Henrik. [...] (WPN11: Katarina av Aragon)

'Am 18. Februar 1516 gebar sie [Katharina] eine Tochter, die Maria (Mary) getauft wurde [...] . Ein männlicher Thronerbe war für Heinrich [VIII] außerordentlich wichtig.'

Im Abschnitt 1 wurde tentativ angenommen, dass nicht generische ISDPs im Norwegischen tendenziell seltener in Subjektfunktion und dementsprechend auch seltener in Vorfeldposition verwendet werden als im Deutschen und dass diese Tendenz auch auf Wiki-NO gegenüber Wiki-DE zutrifft (H1'). Wie die Tabellen 5 und 6 zeigen, spricht die Verteilung der drei ISDP-Kategorien auf die in unseren Stichproben vorkommenden Vorfeldsubjekte zumindest nicht gegen diese Hypothese: Spez-ISDP sind in der Wiki-DE-Auswahl deutlich frequenter als in der Wiki-NO-Auswahl (32,9\% gegenüber 22,1\% für IndefN, 42,2\% gegenüber 34,4\% für IndefXN). Ähnliches gilt für die insgesamt viel selteneren unspezifischen ISDPs (9,2\% versus 0,3\% für IndefN, 9,9\% versus 4,7\% für IndefXN). Umgekehrt sind generische/generalisierende ISDPs in der Wiki-NO-Auswahl stärker vertreten als in der Wiki-DE-Auswahl (77,6\% gegenüber 57,9\% für IndefN, 63,4\% gegenüber 48,3\% für IndeXN). 
Tab. 5: Lesarten von Vorfeld-ISDPs in Subjektfunktion - Wiki-DE-Stichproben

\begin{tabular}{lrrrrrr}
\hline $\begin{array}{l}\text { Strukturtyp } \\
\text { Interpretationstyp }\end{array}$ & IndefN & \multicolumn{3}{c}{ IndefXN } & \multicolumn{3}{c}{ Insg. Indef(X)N } \\
& Anzahl & $\%$ & Anzahl & $\%$ & Anzahl & $\%$ \\
\hline Spez-ISDP & 260 & 32,9 & 320 & 54,7 & 580 & 42,2 \\
Unspez-ISDP & 73 & 9,2 & 58 & 9,9 & 131 & 9,5 \\
Gen-ISDP & 458 & 57,9 & 207 & 35,4 & 665 & 48,3 \\
Insgesamt & 791 & 100 & 585 & 100 & 1.376 & 100 \\
\hline
\end{tabular}

Tab. 6: Lesarten von Vorfeld-ISDPs in Subjektfunktion - Wiki-NO-Stichproben

\begin{tabular}{lrrrrrr}
\hline Strukturtyp & IndefN & & IndefXN & \multicolumn{3}{c}{ Insg. Indef(X)N } \\
Interpretationstyp & Anzahl & $\%$ & Anzahl & $\%$ & Anzahl & $\%$ \\
\hline Spez-ISDP & 196 & 22,1 & 339 & 50,8 & 535 & 34,4 \\
Unspez-ISDP & 3 & 0,3 & 31 & 4,7 & 34 & 2,2 \\
Gen-ISDP & 689 & 77,6 & 297 & 44,5 & 986 & 63,4 \\
Insgesamt & 888 & 100 & 667 & 100 & 1.555 & 100 \\
\hline
\end{tabular}

Wie aus Tabelle 7 hervorgeht, überwiegen unter den Vorfeld-Objekten der WikiDE-Auswahl Spez-ISDPs wie in (16) (IndefXN) und (30) (IndefN). Bei einem guten Teil der so kategorisierten IndefXN-Belege handelt es sich allerdings um attributiv erweiterte Funktionsverbgefüge oder ähnliche mehr oder weniger feste Wendungen der in (31)-(32) veranschaulichten Art, bei denen sich vielleicht diskutieren lässt, inwieweit die abstrakte Objekt-ISDP tatsächlich einen eigenen Diskursreferenten einführt (vgl. Abschnitt 5.3.2). - Gen-ISDPs sind in Objektfunktion selten; ein Bespiel liegt in (33) vor.

Tab.7: Interpretationen von Vorfeld-ISDPs in Objektfunktion - Wiki-DE-Stichproben

\begin{tabular}{lrrrrrr}
\hline $\begin{array}{l}\text { Strukturtyp } \\
\text { Interpretationstyp }\end{array}$ & IndefN & & IndefXN & \multicolumn{3}{c}{ Insg. Indef(X)N } \\
& Anzahl & $\%$ & Anzahl & $\%$ & Anzahl & $\%$ \\
\hline Spez-ISDP & 72 & 80 & 145 & 87,9 & 217 & 85,5 \\
Unspez-ISDP & 11 & 12,2 & 19 & 11,5 & 29 & 11,4 \\
Gen-ISDP & 7 & 7,8 & 1 & 0,6 & 8 & 3,1 \\
Insg. & 90 & 100 & 165 & 100 & 254 & 100 \\
\hline
\end{tabular}

(30) In der Nachkriegszeit Deutschlands wurden Marken auch von privaten Druckereien hergestellt. Ein Beispiel gibt die Bautenserie 1948. (WPD11/ B05.38607: Briefmarkenbogen) 
(31) Boezinge ist ein Dorf in der belgischen Provinz Westflandern, es gehört zur Stadt Ypern. Eine erste Erwähnung fand der Ort 1119 [...] in einer Chronik, die sich heute im St. Martinskapitel in Ypern befindet. (WPD11/B59.62910: Boezinge)

(32) Wissenschaftlern wie Paul Broca und Carl Wernicke gelang es, Hirnregionen ausfindig zu machen, die mit bestimmten kognitiven Fähigkeiten assoziiert sind. Eine große Rolle spielten dabei die Untersuchung von Patienten mit genau lokalisierbaren Läsionen des Gehirns und damit korrelierten kognitiven und/oder mentalen Ausfällen. (WPD11/N15.29523: Neuronales Korrelat des Bewusstseins)

(33) Die Teiladressierung ist sowohl für Consumer als auch für den Businessbereich möglich. Eine Teiladressierung erkennt man an der freien Formulierung anstelle der Namensnennung (z. B. An die Bewohner des Hauses). (WPD11/T16.48207: Teiladressierung)

Von den insgesamt zehn in der Wiki-NO-Auswahl vorkommenden ISDP-Objekten gehören neun zur Kategorie Spez-ISDP - davon acht in IndefXN-NO wie (17) und (34) - und eines zur Kategorie Unspez-ISDP.

(34) Den første følelsen, som de fleste reisende som besøker det moderne Sparta får, er skuffelse over de antikke restene. En bedre «forestilling» gir den bysantinske Mistra, med sine gressbelagte gater, sine forfalne hus, sine festninger i ruiner og sine flotte kirker. (WPN11/S00.25690: Sparta)

'Das erste Gefühl, das die meisten Reisenden, die das moderne Sparta besuchen, erhalten, ist Enttäuschung über die antiken Reste. Eine bessere „Vorstellung“ gibt die byzantinische Mistra, mit ihren grasbelegten Straßen, ihren verfallenen Häusern, ihren Festungen in Trümmern und ihren schönen Kirchen.'

Zusammenfassend halten wir fest, dass die Verteilung der drei hier unterschiedenen ISDP-Lesarten unter den ISDP-Subjekten der Stichprobenkorpora mit der Hypothese H1', dass indefinite Nominalphrasen in Wiki-DE einen größeren Anteil an den nominal besetzten Vorfeldern haben als in Wiki-NO, besser verträglich ist als mit der alternativen Hypothese H1, dass Wiki-DE und Wiki-NO sich in dieser Hinsicht gleich verhalten. Wie schon im Abschnitt 1 bemerkt wurde, müssen die festgestellten Unterschiede bezüglich der Häufigkeit von nicht-generischen Subjekten jedoch keine tieferen sprachlichen Gründe haben: Sie können auch darauf beruhen, dass Artikeltypen, die partikuläre Sachverhalte und Gegenstände/Individuen behandeln, und Artikeltypen verallgemeinernder Natur in den beiden 
Auswahlkorpora ungleich verteilt sind. - Bei den Vorfeld-Objekten der deutschen Stichproben fällt die niedrige Frequenz von Gen-ISDPs relativ zu Unspez-ISDPs und vor allem Spez-ISDPs auf.

\section{Informationsstrukturelle und diskursfunktionale Aspekte}

\subsection{Vorüberlegungen}

Die Vorfeldplatzierung einer Konstituente kann bekanntlich unterschiedlich motiviert sein (s. Zifonun/Hoffmann/Strecker 1997: 1576ff.) - und wird in theoretischer Hinsicht unterschiedlich expliziert; vgl. etwa Speyer (2009) und Féry (2007) mit Frey (2004). ${ }^{11}$ Einigkeit scheint jedoch zumindest in folgenden Punkten $\mathrm{zu}$ bestehen:

(i) Das Vorfeld wird gern mit einem sog. 'aboutness'-Topik besetzt, d.h. mit einer Nominalphrase (DP), die „[d]as Element, um das ist im Satz geht“ (Speyer 2009: 333), identifiziert, - „the entity or set of entitities under which the information expressed in the comment constituent should be stored in the C[ommon]G[round] content“ (Féry/Krifka 2008: 126), „the entity about which the sentence under discussion makes a predication“ (Götze et al. 2014: 163). Nominalphrasen, die nicht referenziell und folglich für die Funktion als 'aboutness'-Topik nicht qualifiziert sind, können jedoch auch im Vorfeld erscheinen (Frey 2007).

(ii) Das Vorfeld kann mit einer Konstituente besetzt sein, die einen (prosodisch markierten) kontrastiven Fokus aufweist. Wie jeder Fokus signalisiert der Kontrastfokus ,the presence of alternatives that are relevant for the interpretation of linguistic expressions“ (Féry/Krifka 2008: 125), aber während die Alternativen zum gewöhnlichen „informational“ bzw. „novelty“-Fokus (Riester/Baumann 2013) eine „,anonyme“ (ebd.) Menge bilden, sind die Alternativen im Fall des Kontrastfokus im weiten Sinne kontextuell beschränkt und grundsätzlich identifizierbar. ${ }^{12}$ Vorfeldkonstituenten mit Kontrastfokus,

11 Leider hat die theoretische Diskussion - mit den einschlägigen Publikationen von Speyer als wohltuenden Ausnahmen - zum großen Teil an nicht oder nur noch rudimentär kontextualisierten Sätzen stattgefunden und indefinite Nominalphrasen dabei wenig beachtet.

12 peyer (2009: 332) verwendet die Bezeichnung „Listen-“/“Poset-Element“ für solche „Elemente in einem gegebenen Kontext, die mit anderen Elementen im Diskurs eine natürliche Men- 
deren (Fokus-)Akzent mit dem (Fokus-)Akzent im Satzinnern korreliert (sog. I-Topikalisierung mit Hutkontur (Jacobs 1997 und Büring 2006), bilden nach Féry (2007) eigene Intonationsphrasen.

(iii) Die Vorfeldbesetzung kann (topologisch) markiert sein. Dies ist, grob gesagt, dann der Fall, wenn die im Vorfeld stehende Konstituente im entsprechenden Verberst- oder Verbletztsatz nicht mit dem gleichen pragmatischen (informationsstrukturellen) Effekt die erste Position im Mittelfeld einnehmen könnte. Unmarkiert ist demgegenüber die Vorfeldstellung einer Konstituente, die sozusagen ihren natürlichen Platz an der Spitze des Mittelfeldes hat. Nach Frey $(2007,2010)$ und Féry (2007) ist die Vorfeldkonstituente im zweiten Fall durch 'Formal Movement' - nach dem Prinzip 'das Vorfeld wird im Defaultfall mit der höchsten Konstituente der finiten Verbalphrase besetzt' - ins Vorfeld gelangt, im ersten Fall hingegen durch Bewegung direkt aus einer tieferen strukturellen Position hinaus (,A-bar-Movement').

(iv) Es wird allgemein angenommen, dass topologische Markierung im Sinne von (iii) prosodische Markierung (starke Prominenz) nach sich zieht (Féry 2007; Frey 2010), wenn auch nicht unbedingt Kontrastfokus mit Hutkontur (Frey 2010).

Für meine Thematik - (singularische) indefinite Nominalphrasen im Vorfeld ergibt sich aus (i)-(iv) Folgendes:

(a) Generisch und spezifisch verwendete ISDPs sind nach den obigen Definitionen mögliche 'aboutness'-Topiks, während unsere Unspez-ISDPs zumindest die strengeren Bedingungen von Féry/Krifka nicht erfüllen, da sie keine Referenten etablieren, die gespeichert werden könnten (Frey 2007; ähnlich Götze et al. 2014). Auf prädikativische Vorfeld-ISDPs komme ich im Abschnitt 5.4 zurück.

(b) Die Vorfeldplatzierung prädikativischer ISDPs ist topologisch markiert; das gleiche trifft auf ISDP-Objekte im Vorfeld zu, sofern ihre Position sich nicht durch kombiniertes 'Scrambling' und 'Formal Movement' erklären lässt (vgl. Féry 2007; Frey 2010). Umgekehrt ist die Vorfeldplatzierung von SubjektISDPs meistens topologisch unmarkiert, entsprechend dem Umstand, dass dem Subjekt im Mittelfeld nur unter besonderen Bedingungen ('scrambling') andere Argumente vorangehen können.

(c) Topologisch unmarkierte Vorfeld-ISDPs - d.h. vor allem Vorfeld-ISDPS in Subjektfunktion - können prosodisch unmarkiert oder kontrastiv fokussiert

ge bilden, wobei die anderen Elemente im gegebenen Kontext eine Alternativenmenge zu dem tatsächlich realisierten Element bilden“ (Poset: partially ordered set). 
sein. Bei prädikativischen Vorfeld-ISDPs und den meisten Objekt-ISDPs ist prosodische Markiertheit zu erwarten.

Vorfeld-ISDPs, die als 'aboutness'-Topiks im strengen Sinne dienen, werden weiterhin als (unmarkierte) 'aboutness'-Topiks (A-Topik) oder kontrastive ('aboutness'-)Topiks bzw. Kontrasttopiks (KA-Topik) bezeichnet je nachdem, ob sie (wenn überhaupt) 'normal' prominent oder kontrastiv fokussiert sind. A-Topiks liegen beispielsweise in (35) (Gen-ISDP) und (36) (Spez-ISDP) vor, während (37) ein (generisches) KA-Topik aufweist.

(35) Es gab zwar keine Gleichstellung von Mann und Frau, aber Hammurapi gewährte den Frauen zahlreiche Rechte. Eine Frau durfte selbständig Rechtsgeschäfte abschließen, wie Kauf und Verkauf und Tauschgeschäfte. (WPD11/C00.38365: Codex Hammurapi)

(36) Eine Königin jammert, dass sie kein Kind hat, und gebiert einen Esel. Sie will ihn ersäufen, aber der Vater lässt das fröhliche Kind aufziehen. (WPD11/ D26.16732: Das Eselein)

(37) [...] Fugen baserer seg hovedsakelig på et hovedtema og et sidetema, som [...]. [...] En dobbelfuge har to temaer. (WPN11/F00.54919: Fuge (musikk)) $)^{13}$ 'Die Fuge basiert überwiegend auf einem Hauptthema und einem Nebenthema, die [...]. Eine Doppelfuge hat zwei Themen.'

Praktische Überlegungen mögen dafür sprechen, unspezifische Vorfeld-ISDPs wie in (38) und (39) gleichfalls als Topiks - oder die Vorfeldposition allgemein als eine Topikposition - zu bezeichnen, wie es oft geschieht. Als 'aboutness'-Topiks können sie ohne eine Revision oder Präzisierung dieses etwas verschwommenen Begriffs nicht gelten. Andererseits zeigen Paare wie (38) und (27), hier als (39) wiederholt, dass auch Unspez-ISDPs prosodisch unmarkiert oder kontrastiv fokussiert sein können. Da die semantisch komplizierten Unspez-ISDPs in den folgenden Abschnitten aus Platzgründen unberücksichtigt bleiben müssen, sei die Frage nach ihrem Topikstatus hier dahingestellt.

(38) Der Südweisende Wagen wurde von dem chinesischen Mechaniker Ma Jun (ca. 200-265) erfunden. Es handelt sich dabei um einen Wagen mit einer frühen Version des Differentialgetriebes. Dadurch zeigt eine Figur auf dem Wagen immer nach Süden. [...] Aber es war nur ein Hülle ohne die Mecha-

13 Die Wörter hovedtema, sidetema und dobbelfuge sind im Original durch Kursivierung hervorgehoben. 
nik. Ein Mann musste im Inneren die Figur drehen. (WPD11/Z38.81769: Zu Chongzhi)

(39) Grab 1 gehört zum Typ der Großdolmen. Ein Grabhügel lässt sich nicht ausmachen, eine Grabeinfassung ist heute nicht mehr vorhanden. (WPD11: Großsteingräber bei Schadewohl)

In den obigen Beispielen handelt es sich um Vorfeld-Subjekte. Das Beispielpaar (40)-(41) veranschaulicht, dass auch Objekt-ISDPs (in casu: Gen-ISDPs) im Vorfeld ohne und mit Kontrastfokus vorkommen; vgl. Punkt (b) oben. - Meine Systematisierung wird in Tabelle 8 zusammengefasst.

(40) Die Teiladressierung ist sowohl für Consumer als auch für den Businessbereich möglich. Eine Teiladressierung erkennt man an der freien Formulierung anstelle der Namensnennung (z.B. An die Bewohner des Hauses). (WPD11/T16.48207: Teiladressierung)

(41) Am häufigsten werden die Dihydropyridine verwendet. Diese wirken hauptsächlich blutdrucksenkend durch direkte Erschlaffung der glatten Gefäßmuskulatur der Arteriolen und nur unwesentlich an der Erregungsleitung des Herzens. [...] Die Phenylalkylamine wirken nur gering auf die Blutgefäße, haben dafür aber eine starke Wirkung auf die Schlagkraft und die Erregungsausbreitung des Herzens. [...] Eine Zwischenstellung nehmen die Benzothiazepine ein, die sowohl gefäßerweiternd und damit blutdrucksenkend als auch hemmend auf die Erregungsleitung im Herz wirken. (WPD11/C05.60701: Calciumantagonist)

Tab. 8: Informationsstrukturelle Funktionen von Vorfeld-ISDPs; Terminologie

\begin{tabular}{lll}
\hline & +'aboutness' im strengen Sinne & ? \\
\hline- Kontrast & A-Topik: Bsp. (35), (36), (40) & Bsp. (38) \\
+ Kontrast & KA-Topik: Bsp. (37), (41) & Bsp. (39) \\
\hline
\end{tabular}

Wenden wir uns jetzt der dritten informationsstrukturellen Dimension (neben Topik-Kommentar- und Fokus-Hintergrund-Gliederung), dem sog. Informationsstatus (s. Féry/Krifka 2008; Götze et al. 2007) zu. Hier scheint es sinnvoll, mit Baumann und Riester (Baumann/Riester 2013; Riester/Baumann 2013) zwischen referenzieller und lexikalischer (oder konzeptueller) Gegebenheit/Bekanntheit bzw. Neuheit usw. zu unterscheiden. Spezifisch gebrauchte indefinite Nominalphrasen führen (diskurs- und hörer-)neue Referenten in den Diskurs ein und sind demnach als referenziell neu (R-neu) zu kategorisieren. Generisch gebrauchte 
(definite und indefinite) Nominalphrasen referieren auf 'kinds' - d.h. Entitäten, die unabhängig vom aktuellen Diskurs enzyklopädisch 'vorhanden' sind. Sie können deshalb mit Götze et al. (2014) als (referenziell) zugänglich (R-zugänglich) bezeichnet werden. ${ }^{14}$ Unabhängig vom referenziellen Informationsstatus der ganzen Nominalphrase (DP) kann das (eventuell modifizierte) Kopfnomen oder eher: die vom Kopfnomen denotierte Menge von Entitäten bzw. das entsprechende Konzept - nach Baumann und Riester lexikalisch neu („L-new“) oder in unterschiedlicher Weise lexikalisch zugänglich („L-accessible) oder gegeben („L-given“) sein. Als L-gegeben zählt das Kopfnomen N, wenn zwischen N und einem im Vorkontext erscheinenden Ausdruck N' Identität oder Synonymie oder aber eine (asymmetrische) mereologische Relation i.w.S. (Teil-Ganzes, ElementMenge, Untermenge-Obermenge etc.) besteht mit $\mathrm{N}$ als dem übergeordneten Term; vgl. (35) und (40). N ist L-zugänglich, wenn N umgekehrt (co-)hyponym, meronym (i.w.S.) zu N' oder anderswie assoziativ mit N' verbunden ist (s. Riester/ Baumann 2013: 226) - d.h., wenn eine typische 'bridging'-Relation vorliegt; vgl. (37), (39) und (42) ${ }^{15}$. L-neu ist das Nomen, wenn die Bedingungen für L-Gegebenheit oder L-Zugänglichkeit nicht erfüllt sind; vgl. (36), (39) und (41) (s. jedoch Abschnitt 5.3.2).

(42) Im Zuge des Bevölkerungsaustausches zwischen Griechenland und der Türkei nach dem Griechisch-Türkischen Krieg 1922 musste die griechische Bevölkerung die Stadt verlassen. Ein Großteil zog in die Peloponnes und gründete in der Argolis die Stadt Nea Kios (Neu-Kios). (WPD11/G06.54628: Gemlik) $^{16}$

14 „Definitional generics“ bilden in dieser Hinsicht möglicherweise eine Ausnahme (vgl. Krifka 2012). - Anders als Götze et al. ordnen Riester/Baumann (2013) generischen Nominalphrasen eine eigene Kategorie R-GENERIC zu, die allerdings auch auf gewisse Typen nicht-referierender indefiniter Nominalphrasen angewandt wird. Wir folgen mit der Kategorisierung '(R-)zugänglich' Götze et al. (2014).

15 Von 'bridging' ist normalerweise nur im Zusammenhang mit definiten Nominalphrasen (und anaphorischen Pronomen) die Rede (s. Irmer 2011 für einen neueren Überblick). Auch Riester/ Baumann (2013) reservieren die Kategorie R-bridging für definite Ausdrücke. Irmer weist (2011: 83) jedoch darauf hin, dass indefinite Nominalphrasen auch „indirekt anaphorisch“ sein können.

16 In (42) hat das relationale Kopfnomen der Spez-ISDP ein implizites anaphorisches Argument, das seine Denotation auf eine im Vorgängersatz spezifizierte Menge von Entitäten restringiert: Ein Großteil muss als ‘ein Großteil davon/ein Großteil der Griechen, die Gemlik verließen’ interpretiert werden. 
Nicht-referenzielle indefinite Nominalphrasen einschließlich unserer UnspezISDPs werden von Götze et al. (2014) und Riester/Baumann (2013) im Hinblick auf ihren Informationsstatus unterschiedlich - und, soweit ich es überblicke, nicht ganz konsequent - gehandhabt. Sie sind in Tabelle 9 als R-unsp(ezifisch) kategorisiert.

Tab. 9: Informationsstatus der ISDPs in (35)-(42)

\begin{tabular}{|c|c|c|c|}
\hline $\begin{array}{l}\text { Informationsstatus } \\
\text { Beispiele }\end{array}$ & Referenziell & Lexikalisch & \\
\hline $\begin{array}{l}\text { (35): Eine Frau } \\
\text { (40): Eine Teiladressierung }\end{array}$ & $\begin{array}{l}\text { R-zugänglich } \\
\text { (generisch) }\end{array}$ & L-gegeben & $\begin{array}{l}\text { (Frau-en bzw. Teiladres- } \\
\text { sierung im Vorgängersatz) }\end{array}$ \\
\hline $\begin{array}{l}\text { (37): En dobbelfuge } \\
\text { 'eine Doppelfuge' }\end{array}$ & $\begin{array}{l}\text { R-zugänglich } \\
\text { (generisch) }\end{array}$ & L-zugänglich & $\begin{array}{l}\text { Hyponymie (fuge ‘Fuge' } \\
\text { im Vorkontext) }\end{array}$ \\
\hline $\begin{array}{l}\text { (36): Eine Königin } \\
\text { (41): Eine Zwischenstellung }\end{array}$ & R-neu & L-neu & (s. jedoch Abschnitt 5.3.2) \\
\hline (42): Ein Großteil & R-neu & L-zugänglich & $\begin{array}{l}\text { Untermenge-Obermenge } \\
\text { (die griechische } \\
\text { Bevölkerung im Vorgän- } \\
\text { gersatz) }\end{array}$ \\
\hline (38): Ein Mann & R-unsp & L-neu & \\
\hline $\begin{array}{l}\text { (39): Ein Grabhügel } \\
\text { Eine Grabeinfassung }\end{array}$ & R-unsp & L-zugänglich & $\begin{array}{l}\text { Teil-Ganzes (Grab im } \\
\text { Vorkontext) }\end{array}$ \\
\hline
\end{tabular}

In den folgenden Abschnitten soll die Interaktion zwischen syntaktischen, semantischen und informationsstrukturellen Eigenschaften der Vorfeld-ISDPs etwas eingehender besprochen werden, und zwar getrennt für Gen-ISDPs (5.2), Spez-ISDPs (5.3) und Präd-ISDPs (5.4); Unspez-ISDPs werden, wie oben erwähnt, nicht weiter berücksichtigt.

\subsection{Generisch/generalisierend gebrauchte ISDPs}

Wie wir im Abschnitt 3 (Tab. 5-7) sahen, machen Gen-ISDPs insgesamt knapp die Hälfte unserer deutschen und knapp zwei Drittel unserer norwegischen VorfeldISDPs aus; dabei handelt es sich fast ausnahmslos um Subjekte. Am stärksten vertreten sind Gen-ISDPs unter den einfachen (zweiwortigen, unattribuierten) ISDPs; in den IndefXN-Stichproben kommen sie erheblich seltener vor (s. Tab. 5 und 6). 


\subsubsection{Gen-ISDP-Subjekte}

Einfache Gen-ISDP in Subjektfunktion sind weitgehend unmarkierte A-Topiks (vgl. für „definitional generics“ Krifka 2012). Typische Beispiele sind (22)-(23), (25)-(26) und (35). Das generische 'aboutness'-Topik dient meistens zugleich als Topik auf Diskursebene, insofern es im jeweiligen Vorgängersatz bzw. als Thema des ganzen Artikels eingeführt worden (also L-gegeben) ist und/oder im nachfolgenden Text fortgeführt wird; ${ }^{17} \mathrm{vgl}$. (43)-(44).

(43) Ein Prisma ist ein lichtdurchlässiger Glaskörper dessen Grenzflächen geschliffen und/oder verspiegelt sind. Ein Prisma dient zur Umlenkung von Lichtstrahlen durch Lichtreflexion oder -brechung. (WPD11/D00.66784: Doppelpentagonprisma)

(44) Vicomte (engelsk: Viscount) er en arvelig høyadelig tittel i flere europeiske land. En vicomte er normalt rangert under grever/jarler, og over baroner. (WPN11/V03.47996: Vicomte)

,Vicomte ist ein erblicher hochadliger Titel in mehreren europäischen Ländern. Ein Vicomte steht im Rang normalerweise unter Grafen/Jarlen und über Baronen.'

Die dreiwortigen Gen-ISDPs des vorliegenden Korpus sind gleichfalls überwiegend gewöhnliche 'aboutness'- und zugleich Diskurstopiks; ${ }^{18}$ vgl. (45)-(46).

(45) Einige Patienten leiden mehrmals an akuter Pankreatitis, können sich aber jedes Mal vollständig erholen. Eine akute Pankreatitis kann eine lebensbedrohliche Erkrankung sein, die zahlreiche Komplikationen hervorruft, normalerweise erholen sich Patienten aber von einer akuten Pankreatitis. (WPD11/P01.35948: Pankreatitis)

(46) Epilepsi kjennetegnes av spontane epileptiske anfall grunnet en funksjonsforstyrrelse i hjernens nerveceller. [...] Et epileptisk anfall er uttrykk for en forbigående funksjonsforstyrrelse i hjernen. (WPN11/E00.56096: Epilepsi) 'Epilepsie ist durch spontane epileptische Anfälle aufgrund einer Funktionsstörung in den Nervenzellen des Hirns gekennzeichnet. [...] Ein epilep-

17 Etwa 50\% der deutschen und 40\% der norwegischen Belege für einfache Gen-ISDPs in Subjektfunktion sind „definitional generics“ (Krifka 2012) der Form ISDP + Kopula + ISDP ... , d.h., sie eröffnen sozusagen einen Diskurs über einen generischen Referenten.

18 Ugf. 30\% der deutschen und 20\% der norwegischen IndefXN-Belege sind Kopulakonstruktionen (ISDP + Kopula + NP ...). 
tischer Anfall ist Ausdruck einer vorübergehenden Funktionsstörung im Hirn.'

Dabei können Adjektiv und Nomen, wie in obigen Beispielen, beide L-gegeben sein; eine Variante davon liegt vor, wenn es sich beim 'Attribut' um die Eigenschaftsanapher solch-/slik(-) handelt wie in (47)-(48) (s. auch Abschnitt 5.3.1). ${ }^{19}$ Oder beide können L-neu/L-zugänglich sein, wie in (49)-(50).

(47) Der Begriff Zischlaut (Sibilant) bezeichnet in der Phonetik einen Laut, der mit einem hörbaren Pfeifen oder Zischen einhergeht. Ein solcher Laut gehört in der Regel zu den Frikativen. (WPD11/Z11.57091: Zischlaut)

(48) En spesialist eller spesiallege er en lege som har gjennomført en spesialistutdannelse og blitt godkjent av helsemyndighetene som spesialist innen en medisinsk spesialitet. En slik utdannelse består både av erfaring gjennom arbeid innen feltet på sykehus o.l., kurs og forskning. (WPN11/S01.40111: Spesiallege)

'Ein Spezialist oder Spezialarzt ist ein Arzt, der eine Spezialistenausbildung durchgeführt hat [...]. Eine solche Ausbildung besteht sowohl aus Erfahrung durch Arbeit auf dem Gebiet in Krankenhäusern o.̈̈., Kursen und Forschung.'

(49) Die mehrjährige Pflanze bildet ein etwa 2 bis $8 \mathrm{~cm}$ hohes so genanntes »Hohlkugelkissen«, eine Sonderform des Polsterwuchses. Ein holziges Stämmchen steckt in Felsspalten oder Geröll, von dort gehen strahlig angeordnete, bis zu $12 \mathrm{~cm}$ lange, wurzelnde Äste aus. Gestauchte Partien mit rosettigen Blättern und gestreckte Partien wechseln sich ab. (WPD11/ S05.45848: Steinschmücke)

(50) or en privatbil i DDR måtte man først søke om kjøpstillatelse og så vente rundt femten år for å få den. En svartebørs eksisterte slik at man kunne forkorte ventetiden. En typisk Trabant kostet 10000 mark ny med ventetid og 30000 mark uten ventetid. (WPN11/D00.06378: Den tyske demokratiske republikk)

'Für ein Privatauto in der DDR musste man zuerst eine Kaufgenehmigung beantragen und dann etwa fünfzehn Jahre auf das Auto warten. Ein Schwarzmarkt existierte, so dass man die Wartezeit verkürzen konnte. Ein

19 Die Wortartenzugehörigkeit dieser Wörter ist unklar: Adjektiv oder (Teil des) Determinativ(s)? Wie das Klassifikationssystem von Riester/Baumann (2013) mit solch- usw. zu Rande kommt, ist mir unklar. 
typischer Trabant kostete 10000 Mark neu mit Wartezeit und 30000 Mark ohne Wartezeit.'

Ist das Adjektiv L-neu oder -zugänglich, das Nomen jedoch L-gegeben wie in (51)(53), so sind die Bedingungen für eine Deakzentuierung des Nomens erfüllt, die gern mit einer kontrastiven Deutung des Topiks einhergeht (s. Riester/Baumann 2013; Götze et al. 2014).

(51) Zur Erfüllung widersprechender Anforderungen wie gutes Führungs- und Störverhalten sind gegebenenfalls aufwändigere Regelkreisstrukturen erforderlich. Ein stabiler Regelkreis kann bei Parameteränderungen instabil werden, selbst wenn die einzelnen Bestandteile des Regelkreises für sich genommen stabil sind. (WPD11/R00.82329: Regelkreis)

(52) Doppelpaddel besitzen keinen Knauf. Ein teilbares Doppelpaddel kann jedoch durch zwei Schaftstücke mit Knauf zu zwei Stechpaddel umfunktioniert werden. Stechpaddel, die als Motorboot- und Yachtzubehör angeboten werden, verfügen teilweise über einen zum Fanghaken ausgeformten Knauf. (WPD11/P00.32536: Paddel)

(53) Jernmangelanemi.

En moderat jernmangel kan gi slapphet uten at det foreligger anemi. Jernmangelanemi kan oppstå på fire måter: [...] (WPN11/A00.51882: Anemi) 'Hämoglobinmangelanämie. Ein moderater Hämoglobinmangel kann zu Schlaffheit führen, ohne dass Anämie vorliegt. Hämoglobinmangelanämie kann in vier Weisen entstehen'

Eindeutig als KA-Topik dient eine dreiwortige ISDP mit L-gegebenem Kopfnomen, wenn der Kontext explizite Alternativen zum Adjektiv bietet und Entsprechendes für den Kommentarteil des aktuellen Satzes gilt; vgl. (54)-(55). Die Alternative(n) zur aktuellen ISDP kann/können aber auch weniger explizit ausgedrückt und allein oder zusätzlich mit dem Kopfnomen verbunden sein, wie in (24) (Schwan gegenüber Singvogel).

(54) Eine Haarfärbung wird vorgenommen, um dem Haar andere Farbnuancen zu geben. [...] Die Haarfärbung lässt sich in Bezug auf die Zahl der Haarwäschen nach der Färbung und der jeweiligen Haltbarkeit der Farbänderung in temporäre, semipermanente und permanente Färbungen einteilen. Eine temporäre Haarfärbung verschwindet nach dem ersten Waschen mit einem Haarshampoo. Eine semipermanente Haarfärbung hält circa zwei bis zehn Haarwäschen aus. Eine permanente Färbung übersteht mehr als zehn Haarwäschen. (WPD11/H03.29997: Haarfärbung) 
(55) En fullstendig forbrenning skjer når det er tilstrekkelig med oksygen,[...]. [...] En ufullstendig forbrenning skjer når det er begrenset tilgang på oksygen. [...] (WPN11/F01.50282: Forbrenning)

'Eine vollständige Verbrennung geschieht, wenn genug Sauerstoff vorhanden ist. [...] Eine unvollständige Verbrennung geschieht, wenn der Zugang zu Sauerstoff beschränkt ist.'

Unter den einfachen Gen-ISDP-Subjekten begegnen KA-Topiks eher selten; vgl. (56)-(57) sowie (37). Sie involvieren gern ein L-zugängliches Kompositum, dessen Erstglied die Funktion des differenzierenden Adjektivs der dreiwortigen ISDP übernimmt; vgl. (56).

(56) Die Freien zahlten die islamischen Zakah- und Fitra-Steuern auf geerntetes Getreide und Vieh. Bei Ernteausfall waren mithin keine Steuern fällig. Eine Mindestmenge war immer steuerfrei. (WPD11/D49.01872: Dar Sila)

(57) Ratsstellen erhöhten Zulagen und das Wahlgeschenk um das Doppelte. Ein „Senior“ erhielt 700, der „Subsenior“ 600 Gulden Gehalt, ein „zeitlicher Dekan“ die doppelten Pfründe. (WPD11/F08.55176: Fürstpropstei Berchtesgaden)

\subsubsection{Gen-ISDP-Objekte}

Als Objekt kommen Gen-ISDPs praktisch nur im deutschen Auswahlkorpus vor (s. Tab. 3 und 4), und zwar sehr selten: Von den insgesamt 673 Gen-ISDP-Vorkommen haben lediglich 8 (1,2\%) Objektfunktion (vgl. Tab. 4 und 6). Es handelt sich dabei teils um unmarkierte L-gegebene Topiks wie in (58) (= (33)) und (59), teils um Fälle wie (60) und (61), wo die L-neue oder L-zugängliche Objekt-ISDP einen Kontrastfokus trägt (Bauernsohn vs. Adel, Speiseabteil vs. Bistroabteil).

(58) Die Teiladressierung ist sowohl für Consumer als auch für den Businessbereich möglich. Eine Teiladressierung erkennt man an der freien Formulierung anstelle der Namensnennung (z.B. An die Bewohner des Hauses). (WPD11/T16.48207: Teiladressierung)

(59) Die Größe des Universums, in dem gespielt wird, lässt sich vor Spielbeginn ebenso festlegen, wie der Prozentsatz der Systeme, in der ein Spieler eine Kolonie errichten muss, um das Spiel zu gewinnen. Eine Kolonie kann man auf friedliche Art gründen, indem man ein spezielles Kolonieschiff auf einen besiedelbaren Planeten oder Mond schickt, aber auch kriegerisch, indem man die Kolonie eines anderen Spielers erobert. Theoretisch ist so auf nied- 
rigen Schwierigkeitsgraden ein friedlicher Sieg möglich. (WPD11/ P13.84411: Perry Rhodan -8211; Operation Eastside)

(60) Durch die Heeresreform Ende des 17. Jahrhunderts, zählte im Heer von nun an vor allem die persönliche Tüchtigkeit und nicht mehr die Abstammung. Einem Bauernsohn stand also der Offiziersstand genau so offen wie dem Adel, wenn er ausreichend Engagement für den Staat zeigte [...]. (WPD11/ R24.99352: Rangtabelle)

(61) Auch einzelne andere Straßenbahnbetriebe experimentierten mit Speiseabteilen, wie in Bonn und Hannover, im Dauerbetrieb durchsetzen konnten sie sich nicht. Ein Bistroabteil gibt es heute in manchen Zügen der Stadtbahn in Karlsruhe. (WPD11/S00.56658: Speisewagen)

Die Vorfeldplatzierung des Objekts ist in beiden Falltypen gut motiviert: Als Mittel der direkten Kontextanbindung (s. Speyer 2009) sichert sie im unmarkierten Topikfall (58)-(59) der L-gegebenen, aber formal indefiniten Gen-ISDP die Topikinterpretation, die sie bei der 'normalen' Mittelfeldplatzierung nicht unbedingt erhalten würde, und erleichtert zugleich die Fokuszuordnung zum postfiniten Teil des Satzes. Im Kontrastfall (60)-(61) dient die Vorfeldplatzierung der im unmittelbaren Vorkontext nicht L-gegebenen ISDP zur Verdeutlichung des Kontrastes bzw. des Topikwechsels.

\subsection{Spezifisch gebrauchte ISDPs}

\subsubsection{Spez-ISDP-Subjekte}

Einfache Spez-ISDPs, die mit jeweils 33\% (IndefN-DE) und 22\% (IndefN-NO) (s. Tab. 5 und 6) die zweithäufigste Kategorie der einfachen Subjekt-ISDPs ausmachen, dienen mit ganz wenigen kontrastiven Ausnahmen (7\% der deutschen Belege) als A-Topiks. Sie haben dabei nicht nur den Informationsstatus R-neu, sondern sind - anders als Gen-ISDPs - im typischen Fall auch L-neu; vgl. (62) und (63). L-zugängliche ISDPs wie ein Großteil in (42) und eine Kugel in (64) kommen jedoch auch vor (eine Kugel kann allerdings auch als KA-Topik aufgefasst werden).

(62) Schließlich finden sie eine Spur. Ein Krankenpfleger hat das Gift, mit dem die Opfer einen qualvollen Tod fanden, aus dem Krankenhaus entwendet. Er entwischt Frederike und Marcello und wird später tot aufgefunden. (WPD11/D24.58214: Donna Roma) 
(63) Psyke gikk til et tårn og bestemte seg for at den raskeste måten å komme til underverdenen var å dø. En stemme stoppet henne $i$ siste øyeblikk og fortalte henne en rute som ville giøre at hun kunne komme seg inn og returnere $i$ live. Stemmen fortalte henne også hvordan hun skulle komme seg forbi Kerberos, Charon og de andre farene på ruten. (WPN11/A00.75162: Afrodite)

'Psyche ging zu einem Turm und entschied sich, der schnellste Weg zur Unterwelt sei zu sterben. Eine Stimme stoppte sie in letzter Minute und sagte ihr einen Weg, auf dem sie hinein und wieder heraus könne. Die Stimme sagte ihr auch, wie sie an Kerberos, Charon und den anderen Gefahren auf dem Weg vorbei komme.'

(64) Als sie dort ankamen, entdeckten die Grenzsoldaten die Gruppe und eröffneten das Feuer. Eine Kugel traf Schmiel im Bauchraum. Ein weiterer Flüchtling wurde an der Schulter getroffen. (WPD11/D45.28674: Dorit Schmiel)

Der neue Referent bleibt oft als A-Topik oder zumindest als Diskursreferent im Nachkontext 'erhalten' und wird dabei durch ein anaphorisches Pronomen oder eine definite Nominalphrase wieder aufgenommen, wie in (20)-(21), (36) und (62)-(63); anders als bei generischen ISDPs - s. (43), (58)-(59) - kann die Wiederaufnahme nicht in Form einer identischen ISDP erfolgen. Nicht selten findet jedoch keine Wiederaufnahme des neuen Referenten statt, oder anders gesagt: Der neu eingeführte Referent 'lebt' oft nicht über den aktuellen (Gesamt-)Satz hinaus. Beispiele sind (65)-(67).

(65) Ein schwerer Unfall ereignete sich am 11. Mai 1970, bei dem nach einem Erdrutsch, ausgelöst durch heftigen, zwei Tage andauernden Regenfall, fünf Menschen zu Tode kamen. Ein Eilzug entgleiste und zertrümmerte den letzten Wagen eines entgegenkommenden Personenzuges. [...] (WPD11/ B35.49452: Bahnhof Beckingen (Saar))

(66) Ein wilder Mann verwüstet den Bauern die Ernte. Ein Jäger fängt ihn mit Schnaps, Wein und Bier. Der Herr stellt ihn in einem Käfig im Schloss aus. (WPD11/D31.31875: Der wilde Mann)

(67) Da han [Cortés] vendte tilbake til Mexico i 1529, kjente han ikke landet igjen. En pest hadde utryddet store deler av befolkningen, og de tidligere fruktbare åkerlandskapet var allerede begynt å bli overgrodd, og overalt var det stille, menneskemylderet var borte. (WPN11/H00.09173: Hernán Cortés) 
'Als er [Cortés] 1529 nach Mexico zurückkehrte, erkannte er das Land nicht wieder. Eine Pest hatte große Teile der Bevölkerung ausgerottet, und die früher fruchtbaren Ackerlandschaften fingen an zuzuwachsen, und überall war es still, der Menschenschwarm war weg.'

Speyer (2009: 338f.) stellt anhand eines Korpus von gut 5.000 Gesamtsätzen fest, dass „frische“ Topiks im Vorfeld „mit großer Mehrheit in mindestens dem folgenden Satz das Topik stellen“ und schließt daraus, dass

Topiks, um vorfeldfähig zu sein, eine makrostrukturelle Bedeutung haben müssen, also in weiteren Sätzen im Diskurs das Topik stellen oder zumindest prominent vorkommen müssen. Bei diskursalten Topiks ist das notwendigerweise gegeben, bei diskursneuen Topiks nicht. Gerade bei letzteren ergeben sich Unterschiede: Nur wenn das Topik im folgenden Diskurs weiterhin Salienz beanspruchen kann, ist es vorfeldfähig. (Speyer 2009: 339)

Einen entsprechenden Ausnahmestatus haben 'kurzlebige' ISDP-Topiks, wie sie in (37) und (65)-(67) vorliegen, in Wikipedia-Texten anscheinend nicht (s. weiter Abschnitt 6).

Es sei hinzugefügt, dass die ISDP als A-Topik nicht selten in einem transitiven Satz erscheint, das ein R-gegebenes (oder R-zugängliches) Objekt enthält; vgl. (62)-(64) und (66)-(67). In solchen Fällen wäre nach unseren Ausführungen im Abschnitt 1 zumindest im Norwegischen eher ein Passivsatz zu erwarten; vgl. (63').

(63') [...] I siste øyeblikk ble hun stoppet av en stemme som fortalte henne en rute som ville gjøre at hun kunne komme seg inn og returnere i live. Stemmen/ Den fortalte henne også hvordan hun skulle komme seg forbi Kerberos, Charon og de andre farene på ruten. (WPN11/A00.75162: Afrodite)

'In letzter Minute wurde sie von einer Stimme gestoppt, die ihr einen Weg sagte, auf dem sie hinein und wieder heraus könne. Die Stimme/Sie sagte ihr auch, wie [...].'

Auch bei den dreiwortigen Spez-ISDP-Subjekten handelt es sich oft um L-neue A-Topiks; vgl. (68)-(69).

(68) Nach den Bombardements kam es zu einigen folgenschweren Unfällen. Ein italienischer Fischer zog eine $250 \mathrm{~kg}$ schwere Bombe an Bord. Bei der Explosion der Bombe wurde er getötet und sein Schiff versenkt. (WPD11/ A37.81565: Annemie Fontana)

(69) Etter skolen studerte Kotsojev på Ardon ortodokse seminar. En plutselig sykdom fikk den unge mannen til å forlate seminaret. Han vendte tilbake til 
landsbyen og begynte å skrive korte noveller for aviser i Nord-Kaukasus. (WPN11/A01.05379)

'Nach der Schule studierte Kotsojev am orthodoxen Seminar Ardon. Eine plötzliche Krankheit brachte den jungen Mann dazu, das Seminar zu verlassen. Er kehrte ins Dorf zurück und fing an, kurze Novellen für Zeitungen im Nord-Kaukasus zu schreiben.'

Anders als im Fall der Gen-ISDPs sind KA-Topiks jedoch mit jeweils ca. 45\% (DE) und 33\% (NO) unter den dreiwortigen Spez-ISDPs-Subjekten stark vertreten. Meistens ist dann das Kopfnomen L-gegeben und dementsprechend deakzentuiert, so dass der Kontrast pränominal lokalisiert ist. Es sind hier zwei Hauptvarianten zu unterscheiden. Die eine - seltener vorkommende - entspricht dem typischen Muster generischer KA-Topiks: Das Attribut ist ein L-neues (oder eventuell L-zugängliches) 'normales' Adjektiv in der Grundform, das einem im Vorkontext erscheinenden Antonym/Kohyponym gegenübersteht oder zumindest ein solches stark evoziert; vgl. (70). Das Attribut trägt in solchen Fällen direkt und unabhängig vom Kontext zur Beschreibung des ISDP-Referenten bei.

(70) Am 29. Mai $1912 \mathrm{zu}$ Wien erfolgte für ihn eine unbeschränkte österreichische Anerkennung des Freiherrenstandes, Diplom ausgestellt am 11. Oktober 1912. Eine italienische Anerkennung folgte am 11. Februar 1930. (WPD11/ L38.87737: Levetzow (Adelsgeschlecht))

Weit häufiger erscheinen jedoch in der pränominalen fokussierten Position kontextabhängige Elemente wie weiter-, ander-, neu-, ähnlich-, solch- und Kardinalia (zweit-, dritt- usw. und erst- als 'kataphorisches' Gegenstück), die keine (prototypischen) Adjektive sind (s. zu solch- und ähnlich- etwa (Umbach/Gust 2014), zu ander-Grønn/Sæbø (2012); vgl. auch Adam/Delettres (in diesem Band). Typische Beispiele sind (71)-(76). ${ }^{20}$

(71) Die Route Innerst - Nonsalm wird im Winter und Frühjahr gerne auch mit Skiern oder Schneeschuhen bestiegen, da der Anstieg relativ lawinensicher ist. Ein zweiter Aufstieg führt von Pill oder der Bezirkshauptstadt Schwaz aus fast parallel zum Gipfel. (WPD11/G07.15815: Gilfert)

20 Belegt sind in IndefXN-NO unter anderen annen/- $t$, 'ander-, $n y(t t)$ 'neu-', senere 'später-', tidligere 'früher-', lignende 'ähnlich-', slik(t) 'solch-', tilsvarende 'entsprechend-' und første 'erst-', andre 'zweit-', tredje 'dritt-'. - Umbach/Gust (2014) kategorisieren solch- als Demonstrativum. 
(72) 60 Bomber, B-25 und B-24, mit einer Eskorte von 30 F6F griffen Betio erneut an. [...] Ein Bomber wurde von der Flak getroffen und musste notlanden. Ein weiterer US-Bomber stürzte wegen Mangels an Treibstoff etwa $100 \mathrm{Mei}$ len vor dem Landeflugplatz von Funafuti ins Meer. Alle anderen entluden die Bomben über dem Flugplatz und über dem kleinen Hafen. (WPD11/ L64.53329: Luftangriffe der Schlacht um die Gilbertinseln)

(73) In der Vorbereitung auf die Saison 2002/03 verletzte sich Petráš, spielte sich nach seiner Genesung dann aber in die Stammelf und holte mit Sparta den tschechischen Titel. Eine erneute Verletzung bremste ihn erneut aus, ehe er ab 2004 wieder Stammspieler bei Sparta war, das 2005 erneut tschechischer Meister wurde. (WPD11/M54.57646: Martin Petrá353)

(74) Im Dezember 2003 ereignete sich in der Stadt ein Gasunfall, bei dem 243 Menschen an Vergiftungen starben. Ein ähnlicher Vorfall ereignete sich im März 2006. Damals wurden jedoch tausende Einwohner in Sicherheit gebracht. (WPD11/G13.48373: Gaoqiao)

(75) Karl den stores fødselsdag er antatt å være 1. april 742. [...] En annen dato er gitt $i$ Annales Petariensis, 1. april 747. (WPN11/K00.26408: Karl den store) 'Als Geburtsdatum Karls des Großen wird der 1. April 742 angenommen. [...] Ein anderes Datum wird in den Annales Petariensis gegeben, 1. April 747.'

(76) På nynorsk kom Det nye testamentet i 1899, og hele Bibelen i 1921. En ny utgave kom i 1938 [...]. (WPN11/D00.18954: Det Norske Bibelselskap) 'Auf Neunorwegisch erschien Das alte Testament 1899, und die ganze Bibel 1921. Eine neue Ausgabe erschien 1938.'

Ein pränominales Element dieser Art restringiert die Menge der unter das Kopfprädikat fallenden Entitäten, aus der der aktuelle ISDP-Referent $x$ 'genommen' wird anhand einer Ähnlichkeitsrelation oder einer (eventuell zeitlichen) Ordnungsrelation relativ zu einem im Vorkontext eingeführten (präsupponierten) Element $y$ der gleichen Menge - dem „target“ nach Umbach/Gust (2014). Inhärent kontrastierend sind auch pränominale Komparativformen wie in (77).

(77) Am 12. und 13. Februar jagte die Gruppe einer japanischen Einsatzgruppe hinterher, konnte gegen die schnell laufenden Schiffe jedoch in keine Angriffsposition gelangen. Ein besseres Ziel bot sich dem Boot am 22. Februar, als [...]. (WPD11/U14.85442: USS Flounder (SS-251)) 
Es sei an dieser Stelle bemerkt, dass Baumann/Riester (2013) und Riester/Baumann (2013) Nominalphrasen (DPs) mit abstraktem Kopfnomen allgemein als R-generic einstufen und sich bei R-generischen DPs nicht um den lexikalischen Informationsstatus des Kopfnomens kümmern. Es besteht jedoch ein grundsätzlicher Unterschied zwischen DPs, die wie (73)-(74) auf partikuläre abstrakte Entitäten (Ereignisse, Sachverhalte, Propositionen, s. Asher 1993) referieren, und der generischen/generalisierenden Verwendung solcher DPs, die etwa in (46), (53) und (55) veranschaulicht wird. Um dem Kopf R-neuer abstrakter ISDPs einen angemessenen lexikalischen (konzeptuellen) Informationsstatus zuordnen $\mathrm{zu}$ können, muss dieser Begriff relativ zu Baumann/Riester (2013) und Riester/Baumann (2013) erweitert werden: Es muss, wie in der Ereignissemantik üblich, angenommen werden, dass Verben und Verbalphrasen (i.w.S.) Mengen von abstrakten Entitäten - Ereignisprädikate - denotieren. In (73) etwa ist der ISDP-Kopf wegen dem Vorkommen von sich verletzen im Vorgängersatz L-gegeben und die ISDP selber R-neu, weil eben von zwei verschiedenen Ereignissen dieser Art die Rede ist. In (78) wiederum muss die im Vorgängersatz zitierte Aussage (Proposition) unter dem Begriff 'Sicht' subsumiert werden, um eine kohärente Interpretation der ISDP zu ermöglichen. Es liegen somit in diesen Fällen komplexe ,indirekte Anaphern“ (Schwarz-Friesel 2000; s. auch Irmer 2011) vor.

(78) „[...] Verbrechen sind auch dann Verbrechen, wenn ihm andere Verbrechen vorausgegangen sind.“ (Rede von Peter Glotz 2001) Eine andere Sicht wird wohl überwiegend in der polnischen Politik vertreten. (WPD11/ V00.22549: Vertreibung)

Unter den einfachen Spez-ISDP-Subjekten kommen KA-Topiks, wie oben erwähnt, relativ selten vor. Der Kontrast kann dabei auf dem (L-zugänglichen) Kopfnomen liegen wie in (79)-(80), wo der Kontext Alternativen in Form von Kohyponymen oder Antonymen bereitstellt. ${ }^{21}$

(79) Im Januar 1892 erkrankte er [Snell] an einer Influenza, von der er sich nicht mehr erholen sollte. Zwei seiner Söhne, Otto Snell und Richard August Emil Snell, wurden ebenfalls Psychiater. Eine Tochter war mit dem Psychiater Julius Bartels verheiratet. Ein Enkel war der Philologe und Rektor der Hamburger Universität, Bruno Snell. (WPD11/L57.55708: Ludwig Snell (Psychiater))

21 "'Da das relationale Kopfnomen ein implizites anaphorisches Argument hat ('ein Enkel von ihm', 'ein Nachweis davon'), dient die ISDP auch in diesen Fällen als indirekte Anapher, nur liegt die Anaphorizität hier nicht am pränominalen Teil sondern am Kopfnomen selber. 
(80) 1719 wurde zum ersten Mal die Vermutung, das Wasser stamme aus der Donauversickerung, im Buch von F. W. Breuninger geäußert. Ein Nachweis gelang jedoch erst am 9. Oktober 1877, als [...]. (WPD11/A02.46778: Aachtopf)

Es kann sich jedoch auch um eine ISDP mit L-zugänglichem oder L-gegebenem Kopfnomen $X$ handeln, der eine dreiwortige ISDP der Form ein (anderes/weiteres/ ...) $X$ (s.o.) oder das andere $X$ im Nachkontext gegenübersteht. In dem Fall kann oder muss der Akzent - sozusagen kataphorisch - auf dem Artikel liegen; vgl. (81)-(83) und Ein Bomber in (72) 22

(81) Det er tre asfalterte veier i Wangdue Phodrang dzongkhag. Hovedveien vest-øst kommer inn i fylket ved Dochu La-passet. En sidevei går nordover fra Wangdue Phodrang til Punakha dzong [...]. En annen sidevei tar av sydover fra hovedveien øst for fylkeshovedstaden og går til klosteret Phobji og til Gangteydalen [...]. (WPN11/W01.46300: Wangdue Phodrang) 'Es gibt drei asphaltierte Straßen in Wangdue Phodrang dzongkhag. Die west-östliche Hauptstraße kommt beim Dochu La-Pass rein. Eine Nebenstraße geht nach Norden von Wangdue Phodrang bis Punakha dzong. Eine andere Nebenstraße biegt östlich der Kreishauptstadt in Richtung Süden ab und $[\ldots]$ '

(82) Das Kunstwerk wird aus zwei unabhängigen treppenartigen Flügeln aus einem satten und dunklen cyanfarbigem Polyester mit einer Gesamtbreite von sechs Metern gebildet. Ein Flügel liegt am Boden, der andere ist aufrecht aufgestellt. (WPD11/A37.81565: Annemie Fontana)

(83) 1944 entschließt er sich, mit Chen und Lee und den 60 Kindern auf einen 700 Meilen langen Marsch „die Seidenstraße hinauf“ bis an den Rand der Wüste Gobi zu gehen, unter anderem über das Gebirge Liupan, und das im kältesten Winter seit 20 Jahren. Ein Kind erhängt sich, eines fällt Kampfhandlungen zum Opfer, ein weiteres wird von einem Wagen erschlagen. (WPD11/D41.15633: Die Kinder der Seidenstraße)

22 Es sei dahingestellt, ob ein(-) in solchen Fällen eher als Zahlwort zu kategorisieren ist. 


\subsubsection{Spez-ISDP-Objekte}

Unter den Objekten im deutschen Auswahlkorpus machen die als spezifisch kategorisierten ISDPs insgesamt 85,5\% aus (Tab. 7). Auf konkrete Entitäten referierende A- bzw. KA-Topiks, wie jeweils in (84) und (85), bilden dabei den Ausnahmefall.

(84) Angeblich gilt sein Lächeln ihrem Dekolleté. Einem Erzbischof soll dieses Dekolleté einst als zu üppig ausgestattet aufgefallen sein, so dass er Steinmetze beauftragte, den Busen abzuflachen. (WPD11/Q09.97262: Queso Tetilla)

(85) Er interpretiert bekannte Songs (Pop-Classic, leichte Klassik, Arien und Musical) und auch eigens für ihn geschriebene Titel. Einem breiteren Publikum begegnete er bei seinen Auftritten bei Art on Ice, [...]. (WPD11/ P50.92724: Patrick von Castelberg)

Bei den meisten der (insgesamt 72) einfachen ISDPs handelt es sich vielmehr um den nominalen Teil einer festen Verbindung vom Typ eine Ausnahme/einen Schwerpunkt/einen Sonderfall/eine Besonderheit/ein Problem bilden/darstellen, eine Rolle/Sonderrolle spielen, sich einen Namen machen; vgl. (86). Diese Konstruktionen entsprechen diskursfunktional zum Teil Konstruktionen mit prädikativischer Vorfeld-ISDP (s. weiter Abschn. 5.4). Eine zweite, nicht ganz so häufige Variante bilden Funktionsverbgefüge wie eine Anwendung/ein Ende finden, eine Erweiterung erfahren; vgl. (87). Hier entspricht die ISDP-'Topikalisierung' der 'Topikalisierung' einer infiniten Verbform (vgl. Angewandt wird dieser Effekt ...). Die Kategorisierung als Spez-ISDP mag in beiden Fällen insofern problematisch erscheinen, als die Vorfeld-ISDP einem ‘topikalisierten' Prädikatsteil entspricht, sie wird jedoch durch mögliche Diskursfortsetzungen der Form Eine zweite Ausnahme bilden ..., Eine weitere Anwendung findet er ...) unterstützt. Gemäß der im Abschnitt 5.1 vorgeschlagenen Systematik haben wir es demnach mit 'aboutness'Topiks zu tun. Die Vorfeldstellung der ISDP ist in solchen Fällen markiert, sie scheint jedoch nicht automatisch mit einem Kontrastfokus einherzugehen; das heißt, die ISDP dient anscheinend je nach Umständen als A- oder KA-Topik; vgl. (86) und (87). Auch in dieser Hinsicht verhalten sich ISDPs dieser Art wie ,thematic predicates“, die nach Frey (2010: 1427f.) mit und ohne „stress“ bzw. mit und ohne eine „emphatic/contrastive interpretation“ vorkommen.

(86) Da Sheffield United rot-weiß gestreifte Trikots trug übernahm man in Derry diese Farben, die auch heute noch getragen werden. Eine Ausnahme bilden die Jahre zwischen 1956 und 1962. Zu dieser Zeit war Derry wenig erfolg- 
reich und eine Änderung der Trikotfarben könnte, so die Hoffnung des Vorstands, dem Club neues Leben einhauchen. (WPD11/D14.07381: Derry City)

(87) Aufgrund der Wechselwirkung zwischen den Magnetfeldern oder dem Magnetfeld und den elektrischen Ladungen der Objekte wirkt eine weitere Kraft die eine Bahnstörung hervorrufen kann. Eine Anwendung findet dieser Effekt im Bereich der Raumfahrtantriebe: als Mini-Magnetospheric Plasma Propulsion (M2P2). (WPD11/B01.10431: Bahnstörung)

Die (insgesamt 145) dreiwortigen Spez-ISDP-Objekte sind gleichfalls (K)A-Topiks, und auch hier überwiegen abstrakte Kopfnomina. Unabhängig von der Kategorie des Kopfnomens liegt der Kontrastfokus (wenn vorhanden) meistens auf dem pränominalen Element, entsprechend dem typischen Muster der als KA-Topiks dienenden dreiwortigen Subjekt-ISDPs (s. Abschnitt 3.3.1); vgl. (88). Kontextunabhängige Adjektive, die den Akzent nicht automatisch auf sich ziehen, sind jedoch auch verbreitet (eine wichtige Rolle spielen, eine große Bedeutung haben, usw.); vgl. (89).

(88) Amsterdam schaffte es [im 18. Jahrhundert], zum finanziellen Zentrum der Welt zu werden, [...]. Bei seiner Ernennung zum König von Holland am 23. Juni 1806 erklärte Louis Bonaparte Amsterdam zu seiner Hauptstadt. Dies konnte jedoch nicht verhindern, dass Amsterdam in den ersten Jahrzehnten des 19. Jahrhunderts allmählich zu einer „toten“ und verarmten Stadt wurde. Einen neuen Aufschwung erlebte die Stadt erst, als 1876 der Noordzeekanaal eröffnet wurde, der Amsterdam eine Verbindung zur Nordsee und damit mit dem Vereinigten Königreich und den Vereinigten Staaten verschaffte. (WPD11/A00.09268: Amsterdam)

(89) Zahlreiche Entwicklungsprojekte widmen sich der Lösung dieses Problems, doch 2-3 Milliarden Menschen werden von keinem dieser Projekte erreicht. Ein bedeutendes Hindernis stellt die Bindung der Förderung seitens der EU-Entwicklungshilfe an private Wasserversorgungsunternehmen dar. (WPD11/T12.58445: Trinkwasser)

Die wenigen im norwegischen Auswahlkorpus registrierten Spez-ISDP-Objekte kommen bis auf eine Ausnahme in der Drei-Wort-Stichprobe (IndefXN-NO) vor. Sie können den obigen Beobachtungen nichts Neues hinzufügen. Beispiele bieten (17) und (34). 


\subsection{Prädikativische Vorfeld-ISDPs}

Präd-ISDPs machen in beiden Teilkorpora lediglich rund 8\% der zweiwortigen ISDPs aus (Tab. 3 und 4). Diese sind überwiegend von der in (90)-(92) illustrierten Art: Der Kopf der ISDP ist - wie bei den typischen Spez-ISDP-Objekten (s.o.) - ein inhärent oder kontextbedingt relationales Nomen wie Beispiel, Ausnahme, Argument, Schwerpunkt, Besonderheit, Voraussetzung, Theorie (bzw. ein norwegisches Pendant), dessen internes Argument zwar nicht realisiert, aber dem Vorkontext eindeutig zu entnehmen ist. Das heißt, die ISDP wird im Hinblick auf das interne Argument anaphorisch interpretiert - ein Beispiel in (90) im Sinne von ein Beispiel dafür verstanden. Andere, nicht-relationale Kopfnomina kommen jedoch auch vor; vgl. (93). Die ISDP ist meistens prosodisch prominent (akzentuiert), aber trotz ihrer markierten Stellung (s. 5.1) nicht kontrastiv markiert; sie kann aber auch den typischen pränominalen Kontrastfokus aufweisen, wie in (94) und eventuell auch (92).

(90) Bei den Stadt- und Staatstheatern im deutschsprachigen und im osteuropäischen Raum hat sich im 19. Jahrhundert dagegen das Repertoiresystem etabliert. Das künstlerische Personal ist hier nicht nur für einzelne Produktionen verpflichtet, sondern oft für mehrere Jahre fest an einem Haus engagiert. Ein Beispiel ist das Wiener Burgtheater, das manchmal an jedem Wochentag eine andere Aufführung aus seinem Repertoire zeigt. (WPD11/ E23.02112: En-suite-Spielbetrieb)

(91) Som med saltvann, kan også en stor mengde ferskvann kalles fjord når innsjøen er lang og smal, [...] En fjord kan òg være en del av en innsjø, som for eksempel Vestfjorden i Tinn. Et unntak er Tyrifjorden, som består av flere smale fjordarmer, men i seg selv (Storfjorden) er forholdsvis vid. (WPN11/I00.27269: Innsjø)

'Wie bei Salzwasser kann auch eine große Menge Süßwasser fjord genannt werden, wenn der Binnensee lang und schmal ist. [...] Ein Fjord kann auch Teil eines Binnensees sein, wie zum Beispiel der Vestfjord in Tinn. Eine Ausnahme ist der Tyrifjord, der aus mehreren schmalen Fjordarmen besteht, aber an sich (der Storfjord) verhältnismäßig breit ist.'

(92) Historikerne vet lite om helsen til Mozart det siste året. En teori er at han døde brått og uventet, og at det hele kom som et sjokk. (WPN11/W00.02449: Wolfgang Amadeus Mozart)

'Die Historiker wissen wenig über den Gesundheitszustand von Mozart im letzten Jahr. Eine Theorie ist, dass er plötzlich und unerwartet starb.' 
(93) Der Alte Garten wird weiterhin für zahlreiche, nun vorwiegend kulturelle Veranstaltungen genutzt. Ein Besuchermagnet sind die seit 1993 jährlich im Sommer, zunächst im Innenhof des Schlosses und seit 1999 [...] auf dem Alten Garten stattfindenden Schlossfestspiele mit ihren Operninszenierungen. (WPD11/A43.44538: Alter Garten (Schwerin))

(94) Befolkningen lenger vest håpet selvfølgelig at fremrykningen skulle fortsette så de også snart skulle bli befridd. At så ikke skjedde skyldtes nok flere forhold. En ting var vinteren som nærmet seg og ville gjort en fremrykning vanskelig. [...] Avgjørende_var det nok også at en eventuell fremrykning ville ført til at tyskerne hadde tvangsevakuert og svidd av landet helt ned til Trøndelag. (WPN11/H08.42678: Huleboerne i Finnmark)

'Die Bevölkerung weiter westlich hoffte natürlich, dass der Vormarsch sich fortsetzen würde, so dass auch sie bald befreit werden würde. Dass dies nicht geschah, hat sicher mehrere Gründe. Eine Sache war der Winter, der nahte und einen Vormarsch erschwert hätte. [...] Entscheidend war sicher auch, dass [...].'

Satzinitiale Präd-ISDPs müssen als unmarkierte bzw. kontrastive 'aboutness'Topiks - (K)A-Topiks - eingestuft werden. Dabei kann der Topik-Referent allerdings kein Individuum sein, falls die ISDP tatsächlich als Prädikativ und der Artikel folglich, nach der kanonischen Analyse von Kopulakonstruktionen mit indefinitem Prädikativ, als semantisch leer zu analysieren ist (Heim 2011). Alternativ dazu könnte die ISDP als eine ‘echte’ indefinite DP und die Kopularelation als die Identitätsrelation analysiert werden. Als Topik-Referent ist aber in jedem Fall die Menge der vom (eventuell kontextuell gesättigten) Kopfnomen denotierten Entitäten bzw. das entsprechende Prädikat aufzufassen. Über diese Menge wird im fokussierten Kommentarteil des Satzes ausgesagt, dass der Subjektreferent dazu gehört bzw. mit einem Element davon identisch ist. In einem entsprechenden Kopula-Satz mit unmarkierter Wortstellung wird umgekehrt über den topikalen Subjektreferenten als neue Information ausgesagt, dass er unter die vom prädikativischen Kommentarteil denotierten Prädikat fällt; man vergleiche dazu etwa (93) mit (93’), wo Subjekt und Prädikativ den Platz gewechselt haben - mit einem weniger kohärenten oder gelungenen Text als Ergebnis.

(93’) Der Alte Garten wird weiterhin für zahlreiche, nun vorwiegend kulturelle Veranstaltungen genutzt. Die seit 1993 jährlich im Sommer, zunächst im Innenhof des Schlosses und seit 1999 [...] auf dem Alten Garten stattfindenden Schlossfestspiele mit ihren Operninszenierungen sind ein Besuchermagnet. 
Die Spitzenstellung der Präd-ISDP ist m.a.W. diskursfunktional gut begründet: Das durch die ISDP festgelegte Topik ist zwar neu, wird aber durch den Vorkontext evoziert - die ISDP ist 'lexikalisch' (semantisch, konzeptuell) zugänglich (vgl. Abschnitt 5.3.1 und 5.1). So lassen Verallgemeinerungen, wie sie beispielsweise in (91)-(92) präsentiert werden, sowohl Beispiele als auch Gegenbeispiele (Ausnahmen) erwarten, Tatsachen verlangen nach Erklärungen, Wissenslücken nach Theorien, usw. (vgl. Zeevat 2014). Der durch die Präd-ISDP eingeleitete Satz beantwortet mithin zumindest partiell eine vom Vorgängersatz abgeleitete, implizite und untergeordnete „Question under Discussion“ (Onea 2013; s. auch Irmer 2011: 173ff.) - die Frage nach Unterstützung für oder Ausnahmen von einer Generalisierung, die Frage nach einer Erklärung für einen überraschenden Sachverhalt, usw. ${ }^{23}$ Die Antwort ist freilich potenziell unvollständig; sie schließt nicht aus, dass andere Entitäten als das Satzsubjekt unter das Topik-Prädikat fallen könnten. Durch die kontrastive Fokussierung, wie sie in (94) vorliegt, wird die potenzielle Unvollständigkeit in eine faktische - die mit einer neutralen Betonung einhergehende schwache Kontrastivität in eine starke - umgewandelt.

Unter den dreiwortigen Präd-ISDPs, die ein gutes Fünftel der Drei-Wort-Stichproben Indef XN-DE und IndefXN-NO stellen (s. Tab. 3 und 4), sind KA-Topiks viel stärker vertreten als unter den einfachen Präd-ISDPs: Jeweils 50\% (IndefXN-DE) und 40\% (IndefXN-NO) der dreiwortigen Präd-ISDPs enthalten, wie die meisten spezifisch-indefiniten KA-Topiks (s. Abschnitt 5.3), ein indirekt anaphorisches, eng fokussiertes Attribut vom Typ ander-, weiter-, ähnlich-, zweit-, das mit einer Deakzentuierung des Kopfnomens einhergeht. Dadurch wird das Kopfnomen oder genauer: das entsprechende Prädikat als explizit oder implizit (L-)gegeben gekennzeichnet, was in Fällen wie (95) und (96) die Subsumption des im Vorgängersatz beschriebenen Sachverhalts unter das ISDP-Prädikat erzwingt. Letzteres ist, wie wir oben sahen, mit dem Topik des aktuellen Satzes zu identifizieren und erweist sich somit im Endeffekt als Topik auch auf Diskursebene: In den Textausschnitten (95) und (96) ist jeweils von Nebeneffekten (von X) und Unterschieden (zwischen europäischem und amerikanischem Film) die Rede.

(95) Besonders der Turbolader und der Ladeluftkühler werden hiervon negativ beeinflusst, es kann zu Leistungseinbußen und Störungen kommen. Ein weiterer Nebeneffekt ist das Mitreißen von unverbrannten Kraftstoffresten,

23 Man bemerke, dass die markierte Wortstellung bei einem nominalen Satzsubjekt eine Elaborierung der Antwort in Form eines direkt anschließenden Relativsatzes ermöglicht; vgl. (90), (91) und (94). 
insbesondere beim Kaltstart, was zur Ölverdünnung des Motoröls führt. (WPD11/B11.75514: Blowby)

(96) Den europeiske filmen har, sammenliknet med den dominerende amerikanske, rykte på seg for å væra mer liberal hva nakenhet og seksualitet angår, men også mindre liberal når det gjelder vold. En annen forskjell er at europeisk film oftest får finansiell støtte fra staten. (WPN11/E04.33538: Europeisk film)

'Der europäische Film hat, verglichen mit dem dominierenden amerikanischen, den Ruf, liberaler zu sein, was Nacktheit und Sexualität betrifft, aber auch weniger liberal, was Gewalt betrifft. Ein weiterer Unterschied ist, dass der europäische Film am häufigsten finanzielle Förderung vom Staat erhält.'

Die restlichen 50\% bzw. 60\% der dreiwortigen Präd-ISDPs entsprechen in informationsstruktureller Hinsicht den typischen einfachen Präd-ISDPs; das Attribut schränkt lediglich den Umfang des Prädikats (bzw. Topiks) ein. Beispiele bieten (97)-(98).

(97) Guthrie ist heute Teil des Oklahoma City Metroplex. Ein wichtiger Wirtschaftsfaktor ist der Tourismus. Die Stadt beherbergt mehrere Museen, darunter die Four-string Banjo Hall of Fame. (WPD11/G21.17942: Guthrie (Oklahoma))

(98) På slutten av 50-tallet og begynnelsen av 60-tallet var flere flyfabrikanter involvert i VTOL-teknologi under militære kontrakter. En uventet deltaker var Curtiss-Wright Corporation som hadde lagt ned flyproduksjonen i 1952 til fordel for produksjon av flymotorer og propeller. (WPN11/C05.06188: Curtiss-Wright X-19)

'Ende der 50er-Jahre und Anfang der 60er-Jahre waren mehrere Flughersteller unter Militärverträgen an der VTOL-Technologie beteiligt. Ein unerwarteter Teilnehmer war Curtis-Wright Corporation, die die Flugproduktion 1952 zugunsten der Produktion von Flugmotoren und Propellern eingestellt hatte.'

Wie bei den einfachen Präd-ISDPs ist das Kopfnomen der prädikativischen DreiWort-ISDPs meistens abstrakt; Beispiele für Konkreta bieten (98) und (99).

(99) Sein Gedicht, das die Grundlage von Home on the Range wurde, trug zunächst den Titel Oh give me a Home bzw. Oh Give Me a Home Where the Buffalo Roam und wurde wahrscheinlich 1872 geschrieben. Ein weiterer Name ist Western Home. (WPD11/H16.74557: Home on the Range) 


\section{Zusammenfassung und Schlusswort}

Im Abschnitt 1 wurde anhand grundlegender struktureller Unterschiede zwischen Deutsch und Norwegisch für die Hypothese (H1') argumentiert, dass indefinite singularische Nominalphrasen (ISDPs) in Wiki-DE einen größeren Anteil an den nominal besetzten Vorfeldern haben als in Wiki-NO. Diese Annahme ist mit den Ergebnissen der im Abschnitt dargelegten, sehr vorläufigen quantitativen Untersuchung verträglich. Zu ihrer Überprüfung sind jedoch größer und differenzierter angelegte Untersuchungen erforderlich.

Im Abschnitt 2 konnte die Hypothese, dass nominale Objekte im Norwegischen erheblich seltener im Vorfeld erscheinen als im Deutschen (s. FabriciusHansen 2015), für Stichprobenkorpora aus dem deutschen und dem norwegischen Wikipedia-Korpus des IDS bestätigt werden. Die Auswahlkorpora umfassen jeweils ungefähr 900 Zufallstreffer für Sätze, die eine Zwei-Wort-ISDP (Indefinitartikel + Nomen), und Sätze, die eine Drei-Wort-ISDP der Form Indefinitartikel + $\mathrm{X}+$ Nomen im Vorfeld enthalten. In beiden Teilkorpora dienten die ISDPs vorwiegend als Subjekte, 15\% waren jedoch Prädikative in Kopulakonstruktionen; ISDPObjekte machten im norwegischen Teilkorpus lediglich 0,5\%, im deutschen hingegen 13,3\% aus (Tab. 3 und 4, Abschnitt 3).

Im Abschnitt 4 wurden die nicht prädikativischen ISDPs der Auswahlkorpora nach ihren referenziellen Eigenschaften in drei Typen kategorisiert: generischgeneralisierende ISDPs (Gen-ISDP), spezifisch referierende ISDPs (Spez-ISDP) und ISDPs, die weder generisch-generalisierend noch spezifisch zu interpretieren sind (Unspez-ISDPs). Dabei stellte sich heraus, dass die Kategorie Gen-ISDP im deutschen und vor allem im norwegischen Auswahlkorpus unter den einfachen ISDP-Subjekten mit jeweils 58\% und 78\% bedeutend stärker vertreten war als unter den dreiwortigen ISDP-Subjekten (35\% bzw. 45\%), während es sich für die Kategorie Spez-ISDP umgekehrt verhielt (jeweils 33\% bzw. 22\% versus 55\% bzw. 51\%) (Tab. 5 und 6). Die unterschiedliche Verteilung hängt sicherlich u.A. damit zusammen, dass Gen-ISDPs, und zwar insbesondere "definitional generics“ (Krifka 2012), öfter als Spez-ISDPs ‘stabile’ Topiks über längere Textpassagen hinweg bilden und Differenzierungen in Form von Attributen in solchen Fällen meistens nicht aktuell sind (vgl. Abschnitt 5.2 und 5.3).

Abschnitt 5 - der Hauptteil - befasste sich mit der informationsstrukturellen Dimension der Vorfeld-ISDPs unter Ausklammerung der unspezifischen. Hier wurde im Abschnitt $5.1 \mathrm{im}$ Anschluss an Féry/Krifka (2008), Frey (2007), Speyer (2009) und vielen anderen zwischen Vorfeldkonstituenten als neutralen 'aboutness'-Topiks (A-Topiks) und Vorfeldkonstituenten als kontrastiv fokussierten 'aboutness'-Topiks oder „Listenelementen“ (Speyer 2009) (KA-Topiks) unterschieden (Abschn. 5.1). Was den sog. Informationsstatus der Nominalphrasen 
betrifft, wurde die von Baumann und Riester (Baumann/Riester 2013; Riester/ Baumann 2013) vorgeschlagene Differenzierung zwischen einer „referentiellen“ und einer „lexikalischen“ (semantischen, konzeptuellen) Ebene der 'Gegebenheit' bzw. 'Neuheit' (oder 'Zugänglichkeit') übernommen. Mit diesem Instrumentarium wurden in den Unterabschnitten 5.2-4 die generisch-generalisierenden, die spezifischen und die prädikativischen ISDPs der Auswahlkorpora untersucht. Unsere Hauptergebnisse lassen sich wie folgt zusammenfassen.

- Wie zu erwarten war, weisen Gen-ISDPs ein eigenes informationsstrukturelles Profil auf: Sie dienen weitgehend als A-Topiks und zugleich als Topiks auf Textebene, und kommen dementsprechend relativ selten mit differenzierenden Attributen vor; als Vorfeld-Objekte sind sie auch im deutschen Teilkorpus äußerst selten (Abschnitt 5.2).

- Dreiwortige spezifisch gebrauchte ISDPs sind bedeutend häufiger KA-Topiks als zweiwortige, die meistens als neutrale A-Topiks dienen (Abschnitt 5.3.1). In beiden Fällen sind die Spez-ISDPs R-neu. Dabei hat das Attribut einer DreiWort-ISDP am häufigsten den Zweck, den R-neuen Referenten von einem im Vorkontext eingeführten Referenten zu differenzieren, der gleichfalls unter das vom Kopfnomen denotierte Prädikat fällt. Das Kopfnomen ist in solchen Fällen L-gegeben und deakzentuiert. Besonders frequent sind als 'Attribute' implizit anaphorische, inhärent kontrastierende Wörter wie ander-, weiter-, zweit-, solch-, ähnlich- bzw. deren Entsprechungen im No.; sie zeichnen sich für 40-50\% der in dreiwortigen Spez-ISDPs enthaltenen 'Attribute'. Bei den prädikativischen ISDPs finden wir mutatis mutandis das gleiche Muster, allerdings etabliert ein Präd-ISDP, wenn die Kopularelation als Prädikation aufgefasst wird, kein 'Individuum', sondern das vom Kopfnomen denotierte Prädikat als Topik (Abschnitt 5.4). - Die als spezifisch kategorisierten ObjektISDPs sind funktional zum großen Teil den Präd-ISDPs vergleichbar (vgl. eine Ausnahme/ein Problem bilden/darstellen und eine Ausnahme/ein Problem sein), sie konnten hier jedoch keine angemessene Analyse erhalten (Abschnitt 5.3.2).

Insgesamt ist festzustellen, dass die satzinitialen ISDPs in unseren Stichproben, auch wenn wir von den generischen absehen, gar nicht so oft einen (R-)neuen individuellen Referenten unter einem (L-)neuen Prädikat in den Diskurs einführen. Meistens ist die Spitzenstellung auch unabhängig von der Satzgliedfunktion als Mittel der direkten Kontextanbindung gut motiviert: Das Kopfnomen ist meistens nicht (L-)neu, sondern aus dem Vorkontext zu erschließen (L-gegeben), oder es wird zumindest durch den Kontext evoziert (L-zugänglich). In vielen Fällen ist die ISDP auch zumindest implizit anaphorisch. Eine Variante dieser Art liegt in den oben erwähnten dreiwortigen ISDPs mit ander-, weiter- etc. oder einer Kom- 
parativform als 'Attribut', eine andere bilden die vielen intrinsisch oder kontextuell relationalen Kopfnomina mit anaphorisch-elliptischem Argument (ein Enkel, ein Großteil, eine Ausnahme, ein Argument, eine Theorie, usw.). Insofern scheinen die hier präsentierten Beobachtungen die Verallgemeinerung zu unterstützen,

\begin{abstract}
dass eine wichtige Aufgabe des Vorfeldes ist, lokale Kohärenz anzuzeigen. [...] Wenn zwei Sätze maximal kohärent aufeinander folgen, d.h. in einer Continue-Relation zueinander stehen, steht das die Kohärenz herstellende Element (nämlich das Center, oder die Phrase, die das Center enthält) bevorzugt im Vorfeld; der Rezipient kann sich dann sofort darauf einstellen, dass der folgende Satz immer noch Information zu derselben Entität hinzufügt, von der bis jetzt die Rede war. (Speyer 2007: 104)
\end{abstract}

Es ist eine interessante Frage (der ich hier nicht nachgehen kann), inwieweit die auch in unserem Material deutliche Vorliebe kontrastiver Konstituenten („Listenelemente“) für die Vorfeldplatzierung sich aus dem Prinzip der lokalen Kontextanbindung ableiten lässt.

Der Vergleich der deutschen und norwegischen Auswahlkorpora hat keine wesentlichen Unterschiede an den Tag gebracht, außer dass Objekt-ISDPs in den norwegischen Stichproben wie erwähnt einen ausgesprochenen Ausnahmefall darstellen und die Vorfeldstellung der ISDP dementsprechend seltener eine topologisch markierte Erscheinung ist als in den deutschen Stichproben. In der Einleitung wurde angedeutet, dass nicht-generische indefinite Vorfeld-Subjekte im Norwegischen wahrscheinlich stärker vermieden werden als im Deutschen. Es sei abschließend betont, dass diese Annahme durch die im Abschnitt 5 festgestellten Übereinstimmungen im Gebrauch der Vorfeld-ISDPs nicht widerlegt wird: Wir haben nicht untersucht, unter welchen Bedingungen und wie oft die Möglichkeit, eine nicht generische Subjekt-ISDP ins Vorfeld zu stellen, nicht genutzt wird. Und sollte sich herausstellen, dass deutsche und norwegische Wikipedia-Artikel sich in dieser Hinsicht nicht signifikant voneinander unterscheiden, so kann das ein - eventuell durch englischsprachige Artikel mit beeinflusstes - Stilmerkmal dieser spezifischen Textsorte sein.

Abschließend muss konkludiert werden, dass der Gebrauch indefiniter Nominalphrasen in der umfangreichen Fachliteratur zum Thema Informationsstruktur nicht die verdiente Aufmerksamkeit erhalten hat - und dass der Begriff des ('aboutness'-)Topiks an theoretischer Klarheit und praktischer Anwendbarkeit immer noch viel zu wünschen übrig lässt. 


\section{Literatur}

Asher, Nicholas (1993): Reference to abstract objects in discourse. (= Studies in Linguistics and Philosophy 50). Dordrecht: Kluwer.

Askedal, John Ole (1986): On ergativity in Modern Norwegian. In: Nordic Journal of Linguistics 9. S. 25-46.

Braun, Peter (1998): Tendenzen in der deutschen Gegenwartssprache. (= Urban-Taschenbücher 297). Stuttgart: Kohlhammer.

Büring, Daniel (2001): What do definites do that indefinites definitely don't? In: Féry, Caroline/ Sternefeld, Wolfgang (Hgg.): Audiatur vox sapientiae: A Festschrift for Arnim von Stechow. (= Studia Grammatica 52). Berlin: Akademie Verlag. S. 70-99.

Büring, Daniel (2006): Focus projection and default prominence. In: Molnár, Valéria/Winkler, Susanne (Hgg.): The architecture of focus. Berlin: de Gruyter. S. 321-346.

Endriss, Cornelia/Hinterwimmer, Stefan (2009): Indefinites as direct and indirect aboutness topics. In: Zimmermann, Malte/Féry, Caroline (Hgg.): Information structure from different perspectives. Oxford: Oxford University Press. S. 89-115.

Engebretsen, Steinar (2012): Norwegische Satzspaltungen und ihre analogen bzw. nichtanalogen deutschen Entsprechungen: Eine korpusbasierte Untersuchung. In: Deutsche Sprache 40. S. 124-150.

Faarlund, Jan Terje/Lie, Svein/Vannebo, Kjell Ivar (1997): Norsk referansegrammatikk. Oslo: Universitetsforl.

Fabricius-Hansen, Cathrine (2015): Satzinitiale indefinite Nominalphrasen im deutschnorwegischen Vergleich. Eine Vorstudie. In: Adam, Séverine/Jacob, Daniel/Schecker, Michael (Hgg.): Informationsstrukturen im Kontrast. Strukturen, Kompositionen und Strategien: Martine Dalmas zum 60zigsten Geburtstag. S. 62-80.

Fabricius-Hansen, Cathrine/Solfjeld, Kåre (1994): Deutsche und norwegische Sachprosa im Vergleich: Ein Arbeitsbericht. (= Arbeitsberichte des Germanistischen Instituts der Universität Oslo 6). Oslo: University of Oslo.

Farkas, Donka F. (2002): Varieties of indefinites. In: Jackson, Brendan (Hg.): Proceedings from semantics and linguistic theory (SALT) XII. (= Proceedings form Semantics and Linguistic Theory 12). Ithaca, NY: Cornell University. S. 59-83.

Féry, Caroline (2007): The prosody of topicalization. In: Schwabe, Kerstin/Winkler, Susanne (Hgg.): On Information Structure, Meaning and Form. Generalization across Languages. Amsterdam: Benjamins. S. 69-86.

Féry, Caroline/Krifka, Manfred (2008): Information structure. Notional distinctions, ways of expression. In: van Sterkenburg, Piet (Hg.): Unity and diversity of languages. Amsterdam: Benjamins. 123-136.

Frey, Werner (2004): A medial topic position for German. In: Linguistische Berichte 198. S. $153-90$.

Frey, Werner (2007): Some contextual effects of aboutness topics in German. In: Späth, Andreas (Hg.): Interfaces and interface conditions. (= Language, Context, and Cognition 6). Berlin: de Gruyter. 329-348.

Frey, Werner (2010): $\bar{A}$-Movement and conventional implicatures: About the grammatical encoding of emphasis in German. In: Lingua 120. S. 1416-1435.

Glück, Helmut/Sauer, Wolfgang Werner (1997): Gegenwartsdeutsch. 2., überarb. und erw. Aufl. (= Sammlung Metzler 252). Stuttgart: Metzler. 
Grønn, Atle/Sæbø, Kjell Johan (2012): A, the, another: A game of same and different. In: Journal of Logic, Language and Information 21. S. 75-95.

Götze, Michael et al. (2014): Information structure. In: Dipper, Stefanie/Götze, Michael/ Skopeteas, Stavros (Hgg.): Information structure in Cross-Linguistic Corpora. (= Interdisciplinary Studies on Information Structure 7). Potsdam: Universitätsverlag Potsdam. S. 147-187.

Haukås, Åsta/Hoheisel, Minka (2013): Das Vorfeld im argumentativen Text. Eine kontrastive Studie des L1-Norwegischen, des L1-Deutschen und des L2-Deutschen. In: Deutsch als Fremdsprache 50. S. 28-35.

Heim, Irene (2011): Definiteness and indefiniteness. In: Maienborn, Claudia/von Heusinger, Klaus/Portner, Paul (Hgg.). S. 996-1025.

von Heusinger, Klaus (2011): Specificity. In: Maienborn, Claudia/von Heusinger, Klaus/Portner, Paul (Hgg.). S. 1025-1058.

Irmer, Matthias (2011): Bridging inferences. Constraining and resolving underspecification in discourse interpretation. (= Language, Context, and Cognition 11). Berlin etc.: de Gruyter. Jacobs, Jochen (1997): I-Topikalisierung. In: Linguistische Berichte 168. S. 91-133.

Krifka, Manfred (2012): Definitional generics. In: Mari, Alda/Beyssade, Clare/Del Prete, Fabio (Hgg.): Genericity. (= Oxford Studies in Theoretical Linguistics 43). Oxford: Oxford University Press. S. 372-389.

Maienborn, Claudia/von Heusinger, Klaus/Portner, Paul (Hgg.)(2011): Semantics: An international handbook of natural language meaning. 2. Halbband. (= Handbücher zur Sprach- und Kommunikationswissenschaft 33). Berlin: de Gruyter.

Onea, Edgar (2013): Potential questions in discourse and grammar. t.a. in Courant Research in the Semantics Pragmatics Interface CRiSPi. Habilitationsschrift. Courantzentrum „Textstrukturen“, Universität Göttingen. https://sites.google.com/site/edgaroneahomepage/research.

Pitz, Anneliese (2006): The relation between information structure, syntactic structure and passive. In: Lyngfelt, Benjamin/Solstad, Torgrim (Hgg.): Demoting the Agent: Passive, middle and other voice phenomena. Amsterdam: Benjamins. S. 225-248.

Ramm, Wiebke (2011): Satzgrenzenveränderungen in der Übersetzung: Satzverbindung und lokale Diskursorganisation im Norwegischen und Deutschen. Ph.D. Dissertation. Oslo: University of Oslo.

Riester, Arndt/Baumann, Stefan (2013): Focus triggers and focus types from a corpus perspective. In: Dialogue and Discourse 4. S. 215-248.

Schwarz-Friesel, Monika (2000): Indirekte Anaphern in Texten: Studien zur domänengebundenen Referenz und Kohärenz im Deutschen. (= Linguistische Arbeiten 413). Tübingen: Niemeyer.

Speyer, Augustin (2007): Die Bedeutung der Centering Theory für Fragen der Vorfeldbesetzung im Deutschen. In: Zeitschrift für Sprachwissenschaft 26. S. 83-115.

Speyer, Augustin (2009): Das Vorfeldranking und das Vorfeld-es. In: Linguistische Berichte 219. S. 323-353.

Sveen, Andreas (1996): Norwegian impersonal actives and the unaccusative hypothesis. Oslo: University of Oslo.

Umbach, Carla/Gust, Helmar (2014): Similarity demonstratives. In: Lingua 114. S. 74-93.

Zeevat, Henk (2014): Production and interpretation of natural language. (= Current Research in the Semantics - Pragmatics Interface 30). Leiden: Brill. 
Zifonun, Gisela/Hoffmann, Ludger/Strecker, Bruno (1997): Grammatik der deutschen Sprache. (= Schriften des Instituts für Deutsche Sprache 7). Berlin: de Gruyter. 